\title{
Restoration of Dendritic Complexity, Functional Connectivity, and Diversity of Regenerated Retinal Bipolar Neurons in Adult Zebrafish
}

\author{
Timothy E. McGinn, ${ }^{1}$ @Diana M. Mitchell, ${ }^{1}$ Peter C. Meighan, ${ }^{2}{ }^{\circledR}$ Natalie Partington, ${ }^{3}$ Dylan C. Leoni, ${ }^{1}$ \\ Christina E. Jenkins, ${ }^{4}$ Michael D. Varnum, ${ }^{2}$ and $\odot$ Deborah L. Stenkamp ${ }^{1}$ \\ ${ }^{1}$ Department of Biological Sciences, University of Idaho, Moscow, Idaho 83844, ${ }^{2}$ Department of Integrative Physiology and Neuroscience, Washington State \\ University, Pullman, Washington 99164, ${ }^{3}$ Department of Biology, Brigham Young University-Idaho, Rexburg, Idaho 83460, and ${ }^{4}$ Martin Luther University, \\ Halle-Wittenberg, Germany 06108
}

Adult zebrafish (Danio rerio) are capable of regenerating retinal neurons that have been lost due to mechanical, chemical, or light damage. In the case of chemical damage, there is evidence that visually mediated behaviors are restored after regeneration, consistent with recovery of retinal function. However, the extent to which regenerated retinal neurons attain appropriate morphologies and circuitry after such tissue-disrupting lesions has not been investigated. Adult zebrafish of both sexes were subjected to intravitreal injections of ouabain, which destroys the inner retina. After retinal regeneration, cell-selective markers, confocal microscopy, morphometrics, and electrophysiology were used to examine dendritic and axonal morphologies, connectivities, and the diversities of each, as well as retinal function, for a subpopulation of regenerated bipolar neurons (BPs). Although regenerated BPs were reduced in numbers, BP dendritic spreads, dendritic tree morphologies, and cone-bipolar connectivity patterns were restored in regenerated retinas, suggesting that regenerated BPs recover accurate input pathways from surviving cone photoreceptors. Morphological measurements of bipolar axons found that numbers and types of stratifications were also restored; however, the thickness of the inner plexiform layer and one measure of axon branching were slightly reduced after regeneration, suggesting some minor differences in the recovery of output pathways to downstream partners. Furthermore, ERG traces from regenerated retinas displayed waveforms matching those of controls, but with reduced b-wave amplitudes. These results support the hypothesis that regenerated neurons of the adult zebrafish retina are capable of restoring complex morphologies and circuitry, suggesting that complex visual functions may also be restored.

Key words: connectome; development; regeneration; retinal bipolar cell; synapse; zebrafish

Significance Statement

Adult zebrafish generate new retinal neurons after a tissue-disrupting lesion. Existing research does not address whether regenerated neurons of adults successfully reconnect with surrounding neurons and establish complex morphologies and functions. We report that, after a chemical lesion that ablates inner retinal neurons, regenerated retinal bipolar neurons (BPs), although reduced in numbers, reconnected to undamaged cone photoreceptors with correct wiring patterns. Regenerated BPs had complex morphologies similar to those within undamaged retina and a physiological measure of photoreceptor-BP connectivity, the ERG, was restored to a normal waveform. This new understanding of neural connectivity, morphology, and physiology suggests that complex functional processing is possible within regenerated adult retina and offers a system for the future study of synaptogenesis during adult retinal regeneration.

\section{Introduction}

The human retina cannot regenerate neurons that are lost due to retinal disease or trauma, whereas retinas of teleost fish have this

Received Nov. 2, 2016; revised 0ct. 27, 2017; accepted Nov. 3, 2017.

Author contributions: T.E.M., D.M.M., P.C.M., M.D.V., and D.L.S. designed research; T.E.M., D.M.M., P.C.M., N.P., D.C.L., and M.D.V. performed research; T.E.M., D.M.M., P.C.M., N.P., D.C.L., C.E.J., M.D.V., and D.L.S. analyzed data; T.E.M., D.M.M., P.C.M., M.D.V., and D.L.S. wrote the paper. capacity (Stenkamp, 2007; Hamon et al., 2016; Wan and Goldman, 2016). Our understanding of mechanisms underlying teleost retinal regeneration has expanded significantly in the last 
decade largely due to the identification of Müller glia as the stem cell source of regenerated neurons (Fausett and Goldman, 2006; Bernardos et al., 2007; Gorsuch and Hyde, 2014; Lenkowski and Raymond, 2014) and to the use of a versatile animal model, the zebrafish (Danio rero), in addition to previous models for retinal regeneration (Stenkamp, 2007). The potential within mammalian retinas to activate mechanisms that can initiate the Müller glial response has been documented previously (Karl et al., 2008; Ueki et al., 2015; Jorstad et al., 2017), catalyzing efforts toward developing regenerative therapies for human retinal disorders (Ahmad et al., 2011; Hyde and Reh, 2014; Jorstad et al., 2017).

Previously, we documented the restoration of visual function in adult zebrafish subjected to chemical damage of the retina and a recovery period, measuring simple reflexes and place preference behaviors (Sherpa et al., 2008; Sherpa et al., 2014). These results indicated that at least some functional synaptic connections are made within regenerated retina. However, a series of studies using diverse modes of retinal damage has shown that the regenerated adult fish retina displays patterning abnormalities in lamination (Sherpa et al., 2014) and in 2D organization of specific neuronal classes (Hitchcock et al., 1992; Stenkamp et al., 2001). The regenerating adult zebrafish retina also generates supernumerary neurons (Sherpa et al., 2008; Sherpa et al., 2014; Powell et al., 2016), including those that were not lost to damage (Fraser et al., 2013; Powell et al., 2016). Together, these observations suggest that regenerated retinal neurons may be capable of accurate rewiring even in a disrupted microenvironment and despite abnormal cellular patterns. Previous work in regenerated adult goldfish retina showed that individual synaptic terminals of regenerated neurons in the outer plexiform layer (OPL) and inner plexiform layer (IPL) were normal at an ultrastructural level (Hitchcock and Cirenza, 1994) and that regenerated neurons could integrate functionally with undamaged neurons in the lateral dimension via gap junctions (Hitchcock, 1997). However, the degree to which regenerated retinal neurons in adult fish establish a normal retinal connectome to the correct type and number of synaptic partners and whether regenerated neurons attain normal morphologies that support complex functions remain undetermined.

Recently, D'Orazi et al. (2016) discovered that selected subpopulations of regenerated retinal bipolar neurons (BPs) of the growing larval zebrafish retina established essentially normal morphologies and regained connectivity patterns with their undamaged synaptic partners, displaying some minor errors. Therefore, it remains unclear whether regenerated retinal neurons can also restore appropriate morphologies and connectivity patterns with synaptic partners that have also regenerated, if they can do so within a disturbed environment of more profound retinal damage, if physiological connectivities are restored, and if any of these can take place in adult animals.

In the present study we focus upon a morphologically diverse subpopulation of retinal BPs that are regenerated after a chemical

We thank Dr. S. Kawamura for the sws1:GFP fish, Dr. P. Raymond for the sws2:mCherry fish, and Dr. R. Wong for the nyx::mYFP fish; Ruth Frey, Tyler Lankford, Jared Lambert, Carlos Galicia, and Amber Trost of the Stenkamp laboratory and Ann Norton of the University of Idaho's Optical Imaging Core for technical assistance; Dr. Celeste Brown and Chloe Stenkamp-Strahm for assistance with statistics and data visualization; Dr. David DeWitt for expert contributions to optimization of the ERG recording setup; and Dr. Peter Fuerst for discussion and evaluation of earlier versions of the manuscript. Antibody ZPR1 came from the Zebrafish International Resource Center.

The authors declare no competing financial interests.

Correspondence should be addressed to Deborah L. Stenkamp, Professor, Biological Sciences, 875 Perimeter Drive, MS 3051, University of Idaho, Moscow, ID 83844-3051. E-mail: dstenkam@uidaho.edu.

DOI:10.1523/JNEUROSCI.3444-16.2017

Copyright $\odot 2018$ the authors $\quad 0270-6474 / 18 / 380121-17 \$ 15.00 / 0$ lesion that destroys virtually all inner retinal neurons but spares photoreceptors and Müller glia (Fimbel et al., 2007; Nagashima et al., 2013; Sherpa et al., 2014). Regenerating BPs were therefore challenged to elaborate their dendritic and axonal processes and to find presynaptic partners that were undamaged (cone photoreceptors), as well as postsynaptic partners that were themselves regenerating or had regenerated (amacrine and ganglion cells; Fimbel et al., 2007; Nagashima et al., 2013; Sherpa et al., 2014). Through detailed morphometric analyses of dendritic and axonal attributes and photoreceptor connectome and axon stratification patterns, we conclude that regenerated retinal BPs show largely normal morphologies and connectomes and diversities of each of these. Furthermore, ERG recordings from regenerated retinas showed normal waveform features with reduced amplitude for the b-wave, consistent with functional restoration of photoreceptor-BP connectivities.

\section{Materials and Methods}

Animals and retinal lesioning. All zebrafish were treated in accordance with protocols approved by The University of Idaho and Washington State University Institutional Animal Care and Use Committees and were raised and maintained according to Westerfield (2007) on a 14:10 h light:dark cycle in monitored, recirculating system water. Zebrafish transgenic strains used in this study were nyx::mYFP [two transgenes likely cointegrated: nyctalopin (nyx) promoter driving gal4 and the UAS enhancer element driving MYFP; Schroeter et al., 2006] with YFP expressed in retinal BPs; sws2:mCherry (Salbreux et al., 2012) with mCherry expressed in blue-sensitive cones; and sws1:GFP (Takechi et al., 2003) with GFP expressed in UV-sensitive cones.

The retinas of adult fish (6-16 months old) of either sex were chemically lesioned by intravitreal injection of ouabain to destroy inner retinal neurons selectively while sparing photoreceptors and Müller glia (Maier and Wolburg, 1979; Fimbel et al., 2007; Nagashima et al., 2013; Sherpa et al., 2014). Briefly, fish were anesthetized by tricaine and an incision was made across the cornea and iris with a sapphire knife. Using a Hamilton syringe ( $26 \mathrm{~s}$ ), $0.4-0.6 \mu \mathrm{l}$ of $70 \mu \mathrm{m}$ ouabain in saline was injected into the vitreal chamber of one eye, resulting in an estimated intraocular concentration of $2 \mu \mathrm{M}$. The contralateral eyes of lesioned fish served as uninjected, undamaged controls. Loss of BPs and survival of cones were verified in sectioned retinas obtained from parallel experiments at $3 \mathrm{~d}$ postinjury (DPI) (see Fig. 1) and by viewing retinas of live, anesthetized fish with epifluorescence stereomicroscopy (Nikon SMZ 1500), also at 3 DPI.

Some of the lesioned zebrafish were exposed to bromodeoxyuridine (BrdU), which is incorporated into cells in S-phase and later visualized to confirm that a cell was generated during the period after lesioning. For these experiments, fish were transferred to beakers containing $1 \mathrm{~mm}$ BrdU in system water, with treatment solutions changed every $24 \mathrm{~h}$. Exposures took place from 4-7 DPI or from 7-12 DPI.

Tissue processing and immunocytochemistry. Fish were sacrificed with tricaine and eyes enucleated. To obtain retinal cryosections, whole eyes were fixed in phosphate-buffered, $\mathrm{pH} 7.4,4 \%$ paraformaldehyde containing $5 \%$ sucrose for $1 \mathrm{~h}$. Eyes were washed, cryoprotected, embedded, and frozen in a 1:2 solution of Tissue-Tek optimal cutting temperature embedding medium (Sakura Finetek) and phosphate-buffered, 20\% sucrose (Sherpa et al., 2008; Sherpa et al., 2011; Sherpa et al., 2014). Eyes were cryosectioned at 5 or $20 \mu \mathrm{m}$.

To obtain whole, flat-mounted retinas, enucleated eyes were perforated through the cornea with a 27-gauge needle and the hole expanded with dissecting scissors. The lens was removed through the incision and then the sclera and retinal pigment epithelium (RPE) were peeled away with forceps to free the retina. Retinas were rinsed in cold HEPES or PBS and four radial incisions were made to facilitate flattening the retina. Retinas were then fixed for $1 \mathrm{~h}$ at room temperature in phosphatebuffered $4 \%$ paraformaldehyde containing $5 \%$ sucrose. For BrdU detection, fixed whole retinas were pretreated with a $1: 1$ mixture of $4.0 \mathrm{~N}$ 
$\mathrm{HCl} / \mathrm{PBST}$ for $45 \mathrm{~min}$, then washed in PBS containing $1 \%$ Tween and 1\% Triton X-100 before antibody staining.

For immunohistochemistry, 5 and $20 \mu \mathrm{m}$ sectioned retinas were rinsed with PBS with $0.5 \%$ Triton (PBST) and then blocked for $1 \mathrm{~h}$ at room temperature with $20 \%$ normal goat serum and $0.1 \%$ sodium azide diluted in PBST. Sections were stained with primary antibodies diluted in antibody dilution buffer (PBST, $1 \%$ normal goat serum, and $0.1 \%$ sodium azide). Sections were incubated overnight at $4^{\circ} \mathrm{C}$, washed 3 times with PBST, and incubated with secondary antibodies diluted in antibody dilution buffer and $4.25 \mu \mathrm{M}$ DAPI overnight at $4^{\circ} \mathrm{C}$. Whole retinas were stained with primary antibodies in antibody dilution buffer for 1-2 weeks at $4^{\circ} \mathrm{C}$, washed 3 times with PBST, and then incubated with secondary antibodies diluted in antibody dilution buffer and $4.28 \mu \mathrm{m}$ DAPI at $4^{\circ} \mathrm{C}$ for another week. Slides or retinas were washed $3 \times 30 \mathrm{~min}$ in PBS before mounting.

Primary antibodies used in this study were as follows. ZPR1 is a mouse monoclonal that labels cone arrestin3a, staining both members of the red- and green-wavelength-sensitive double cone pair (ZIRC, 1:200; Renninger et al., 2011). Anti-protein kinase $\mathrm{C} \alpha(\mathrm{PKC} \alpha)$ is a rabbit polyclonal antibody originally produced to target the $\mathrm{C}$ terminus of human PKC $\alpha$, mapped at aa 372-672. PKC $\alpha$ labels a subpopulation of BP neurons (SC-10800, 1:200, Santa Cruz Biotechnology; Suzuki and Kaneko, 1990). Anti-synaptic vesicle 2 (SV2) is a mouse monoclonal antibody that labels synaptic terminals (1:2000, Developmental Studies Hybridoma Bank; Yazulla and Studholme, 2001). Anti-BrdU is a rat monoclonal antibody (1:200; Invitrogen). Secondary antibodies used in this study were donkey anti-mouse Dylight 649 (1:200; Jackson Laboratories), donkey anti-rabbit Cy3 (1:200; Jackson Laboratories), and donkey anti-rat Alexa Fluor 647 (1:200; Thermo Scientific).

Imaging and analysis. Sectioned, stained retinas were mounted in Fluoromount G (Southern Biotech) or Vectashield Hardset (Vector Laboratories) with number 1 coverslips (Fisher Scientific). Some sections were imaged using a $20 \times 0.75$ numerical aperture (NA) air objective or a $60 \times, 1.42$ NA oil-immersion objective on an Olympus FV1000 confocal microscope. Another set of sections was imaged with an Andor Zyla 5.5 sCMOS camera connected to a Nikon Ti inverted microscope with a Yokogawa spinning disk using a $20 \times 0.75 \mathrm{NA}$ air objective, a $60 \times 1.40$ $\mathrm{NA}$, or $100 \times 1.45 \mathrm{NA}$ oil-immersion objective.

Whole retinas were flattened and mounted on microscope slides in Fluoromount G or Vectashield Hardset with number 1 coverslips with ganglion cell layer (GCL) facing the coverslip. Electrical tape was used as a spacer between the coverslip and slide. Whole, flat-mounted retinas were imaged with the Andor/Nikon system described above using a $20 \times$ air $0.75 \mathrm{NA}$ objective or a $60 \times 1.2 \mathrm{NA}$ water-immersion objective with Immersol W 2010 (Zeiss). Using the $60 \times$ lens, multiple image stacks were collected using $0.3 \mu \mathrm{m} z$-steps through the entire thickness of the retina. Each stack was $114.62 \mu \mathrm{m}$ wide by $164.88 \mu \mathrm{m}$ high $(1028 \times 1522$ pixels), resulting in a scale of 9.23 pixels/ $\mu \mathrm{m}$. Most image stacks were collected from regions outside of the larval remnant surrounding the optic nerve head (Allison et al., 2010), and avoiding the most peripheral regions. Images of whole retinas stained with $\mathrm{BrdU}$ were captured using a $60 \times 1.40$ NA oil objective.

Image stacks were analyzed in Fiji software (version 1.51d; RRID: SCR_002285; Schindelin et al., 2012). Images were gathered from multiple locations across the retina. Generally, most confocal image stacks were taken near the central retina as well as several adjacent areas. Additional confocal image stacks were taken in the midperiphery, where nyx: $m Y F P^{+}$cells were clearly isolated. Selected nyx::mYFP ${ }^{+}$cells were traced using the Simple Neurite Tracer (SNT) plugin (Longair et al., 2011). Although some sampled nyx::mYFP ${ }^{+}$cells were clearly isolated, others had near or overlapping neighbors. These neurons were included in this study to ensure that multiple types and locations of nyx: $m Y F P^{+}$cells were sampled and traced. Dendritic spread was measured using the ellipse method, in which an ellipse was drawn around the perimeter of the dendritic tips and the area of the ellipse recorded (Li et al., 2012), and using a convex polygon, in which a polygon was drawn around the outermost tips of the dendrites and the area was recorded (Kraft et al., 2006). Feret's diameter was measured from the ellipses generated to measure dendritic spread. These ellipses represented a "collapsed" dendritic tree into a single plane parallel to the OPL.

Sholl analysis was carried out on traced BP dendrites and axons. Using the Sholl analysis option in SNT, data for each dendrite and axon were separately exported as column separated value files (.CSV) that contained the number of intersections at each radial distance from the center. These .CSV files were batch analyzed using the Sholl Analysis Plugin (version 3.6.12) packaged with Fiji (Ferreira et al., 2014). For dendrites, the point at which the primary dendrite branched to generate the dendritic network was considered the center point. The center point used for the axons was at the furthest stratification point from the soma and where the axon initially branched.

To identify connections of cones to nyx::mYFP BPs, original .ND2 raw images were imported into Fiji (Schindelin et al., 2012). Dendrites of $n y x:: m Y F P$ BPs were each traced separately in SNT. Traced dendrites were filled in using the "fill" option and independent stacks for each dendrite were generated. These stacks were remerged into the original stack as separate channels. Only the channels that included ZPR1 ${ }^{+}$ cones, sws2:mCherry (blue-sensitive) cones, and the traced BP neuron were viewable. A partial $Z$-projection was generated such that the projected image only contained the synaptic pedicles of the cones and the dendritic field of the BP cell being analyzed. Using the region of interest (ROI) tool in Fiji, the synaptic pedicles of the sws2:mCherry cones and the $\mathrm{ZPR} 1{ }^{+}$cones were outlined as separate colors. In many cases, the identity of $\mathrm{ZPR}^{+}{ }^{+}$pedicles as belonging to an $\mathrm{RH} 2$ versus LWS (green-sensitive vs red-sensitive) cone could be determined from the image because this marker more strongly labels red-sensitive cones. In addition, cone identities could be established based upon their location within the cone mosaic of the zebrafish retina (Allison et al., 2010; Li et al., 2012). Each dendritic tip that connected with an identified cone pedicle was recorded, as well as the total number of dendritic tips for each BP cell analyzed.

Using Fiji, individually traced neurons were cropped and then resliced using the "Stacks Reslice [/]" tool. This generated images with radial views of the retina such that the IPL could be subdivided into six equivalent strata (Connaughton et al., 2004), which was possible when laminar fusions were not extensive. Axon stratification patterns of individual BP cells were determined by recording the stratum or strata that contained axon terminals. Some neurons were visualized by using Fiji for space filling and then exporting into 3DS Max for surface rendering.

The thickness of the IPL and of the overall length of the BPs were measured with the line tool in Fiji using PKC $\alpha$-stained cryosections. For IPL thickness, the line was drawn from the innermost portion of the $\mathrm{DAPI}^{+}$or SV2 ${ }^{-}$portion of the inner nuclear layer (INL) to the most distal tips of PKC $\alpha^{+}$axons in the deepest IPL stratum. For overall BP length, the line was drawn from the most distal point of the primary dendrites of $\mathrm{PKC} \alpha{ }^{+}$BP neurons to the deepest tips of the primary axons. Each measurement was taken from three different locations of one radially oriented section and averaged for each of three eyes per condition.

Numbers of BP neurons were quantified from PKC $\alpha$-stained retinal cryosections of $n y x:: m Y F P$ fish ( $n=3$ per condition for this analysis) by counting PKC $\alpha^{+}$, DAPI $^{+}$cell bodies, $n y x:: m Y P^{+}{ }^{+}$DAPI $^{+}$cell bodies, and colabeled cell bodies across $5 \mu \mathrm{m}$ radial sections imaged at $20 \times$ and sampled between the larval remnant (Allison et al., 2010) and the extreme periphery. To avoid oversampling due to split nuclei, we counted from every fifth section. Percentage recovery at $60 \mathrm{DPI}$ was calculated as follows:

$$
\frac{\# B P s @ 60 D P I-\# B P s @ 3 D P I}{\# \text { ControlBPs }-\# B P s @ 3 D P I} \times 100
$$

Experimental design and statistical analysis. Quantification of $\mathrm{PKCa}^{+}$ and $n y x:: m Y F P^{+}$BPs was done for $n=3$ control, $n=3$ DPI, and $n=60$ DPI eyes. Sampling depth for each neuron-specific parameter and characteristic ranged from 11-30 individual neurons per condition, with neurons sampled from 10 different undamaged eyes and 10 different eyes collected at 60 DPI. Data were imported into R Studio (version 0.99.903; R Project for Statistical Computing, RRID:SCR_001905) using R (version 3.4.1; CRAN, RRID:SCR_003005) for statistical analysis. Welch's two-sample $t$ tests, paired $t$ tests, and Kruskal-Wallis tests were used for parametric and most nonparametric data, respectively. Kruskal-Wallis 

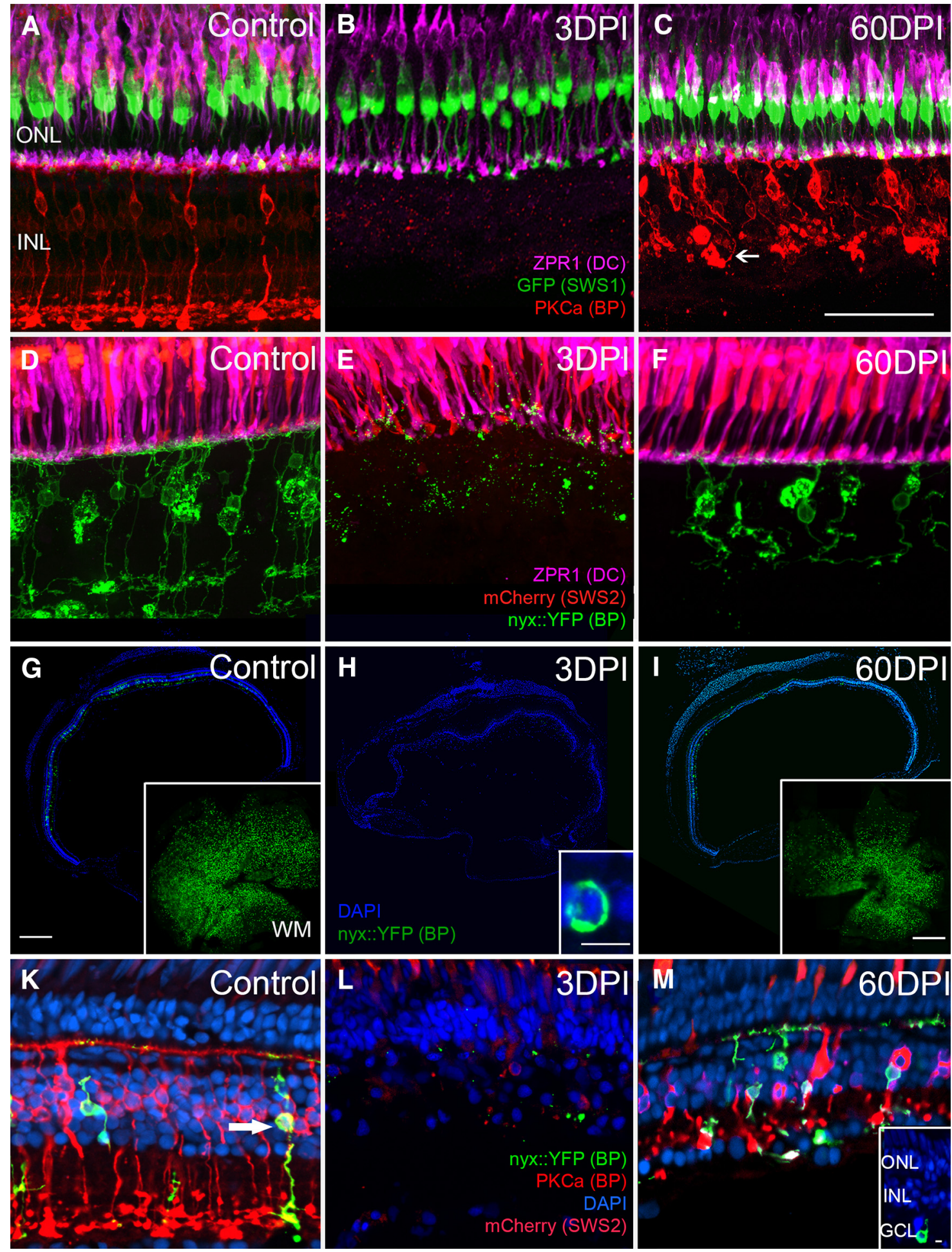

Figure 1. Intravitreal $2 \mu \mathrm{m}$ ouabain destroys retinal BPs, spares cone photoreceptors, and permits regeneration of $\mathrm{BPs}$. $A$, Undamaged retina showing ZPR1 ${ }^{+} \mathrm{DCS}, \mathrm{UV}(\mathrm{SWS1}) \mathrm{cones}$, and PKC $\alpha{ }^{+}$ BPs. $B$, Damaged retina at 3 DPI showing loss of BPs and persistence of cones. $C$, Damaged and then regenerated retina at $60 \mathrm{DPI}$ showing regenerated PKC $\alpha^{+} \mathrm{BPS}$; arrow indicates a BP axon lacking the typical, straight trajectory. $\boldsymbol{D}$, Undamaged retina showing ZPR1 ${ }^{+} \mathrm{DCS}$, blue (SWS2) cones, and nyx::mYFP ${ }^{+}$BPs. E, Damaged retina at 3 DPI showing loss of BPs and persistence of cones. $\boldsymbol{F}$, Damaged and then regenerated retina at 60 DPI showing regenerated nyx::mYFP ${ }^{+}$BPs. $\boldsymbol{A}-\boldsymbol{F}$ show $20 \mu \mathrm{m}$ sections. $\mathbf{G}-\boldsymbol{I}$, Stitched images of entire $5 \mu \mathrm{m}$ sections of control retina $(\boldsymbol{G})$, damaged retina $(\boldsymbol{H})$, and regenerated retina $(\boldsymbol{I})$ showing normal distribution of nyx::mYFP ${ }^{+}$cell bodies, loss of these cells at $3 \mathrm{DPI}$, and restoration in reduced numbers at $60 \mathrm{DPI}$. Insets in $\boldsymbol{G}$ and $\boldsymbol{I}$ show low-magnification views of whole-mounted control and regenerated retinas, respectively. Inset in $\boldsymbol{H}$ shows a high-magnification view of one example of the very few nyx::YFP ${ }^{+}$cell bodies that remain at 3 DP l lacking any cellular processes. $\boldsymbol{K}-\boldsymbol{M}$, Sections $(5 \mu \mathrm{m})$ of control retina $(\boldsymbol{K})$, damaged retina $(\boldsymbol{L})$, and regenerated retina $(\boldsymbol{M})$ showing minimal or no colabeling of $n y x:: m Y F P$ with PKC $\alpha$ in all preparations. Arrow in $\boldsymbol{K}$ shows one such colabeled BP. Inset in $\boldsymbol{M}$ shows a lower-magnification view of one example of the few nyx:: $m Y F P^{+}$cell bodies displaced in the GCL of a regenerated retina. Scale bar in $\boldsymbol{C}$ applies to $\boldsymbol{A}-\boldsymbol{F}$ and $\boldsymbol{K}-\boldsymbol{M}$ and is $50 \mu \mathrm{m}$; scale bar in $\mathbf{G}$ applies to $\boldsymbol{G} \boldsymbol{I}$ and is $200 \mu \mathrm{m}$; scale bars in insets of $\boldsymbol{H}$ and $\boldsymbol{M}, 5 \mu \mathrm{m}$; scale bar in inset of $\boldsymbol{I}$ applies also to inset of $\boldsymbol{G}$ and is $500 \mu \mathrm{m}$. 


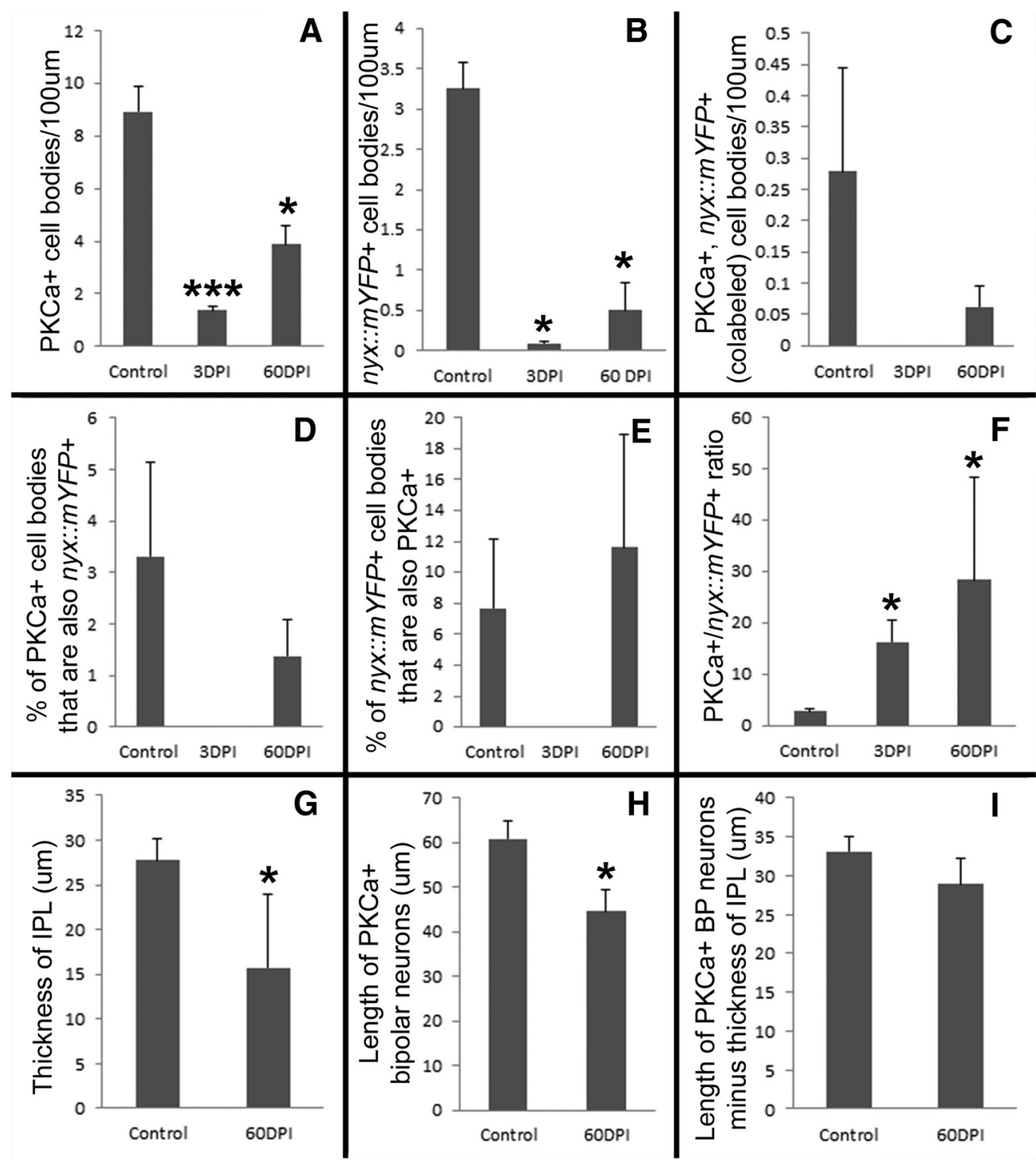

Figure 2. $\quad \boldsymbol{A}-\boldsymbol{C}$, Quantification (mean $\pm \mathrm{SEM})$ of PKC $\alpha^{+}(\boldsymbol{A})$, nyx:::mYFP $(\boldsymbol{B})$, and colabeled cell bodies $(\boldsymbol{C})$ of control retina, damaged retina $(3 \mathrm{DPI})$, and regenerated retina $(60 \mathrm{DPI}) . \boldsymbol{A}, \chi_{(2)}^{2}=$ $7.2 ;{ }^{* * *} p=0.00097$ for 3 DPI versus control; ${ }^{*} p=0.0104$ for 60 DPI versus control (Kruskal-Wallis, Conover's post hoc). $\boldsymbol{B}, \chi_{(2)}^{2}=5.6 ;{ }^{*} p=0.037$ for 3 DPI versus control; ${ }^{*} p=0.045$ for $60 \mathrm{DPI}$ versus control (Kruskal-Wallis, Conover's post hoc). $\boldsymbol{C}, \chi_{(2)}^{2}=3.307, p=0.191$ (Kruskal-Wallis). $\boldsymbol{D}-\boldsymbol{F}$, Proportions colabeled $(\boldsymbol{D}, \boldsymbol{E})$, and ratios $(\boldsymbol{F})$ of PKC $\alpha^{+}$and $n y x \times: m Y F P$ cell bodies. $\boldsymbol{D}, \chi_{(2)}^{2}=$ $3.307, p=0.191$ (Kruskal-Wallis). $\boldsymbol{E}, \chi_{(2)}^{2}=2.9867, p=0.2246$ (Kruskal-Wallis). $\boldsymbol{F}, \chi_{(2)}^{2}=5.422 ;{ }^{*} p=0.038$ for 3 DPI versus control; ${ }^{*} p=0.038$ for 60 DPI versus control (Kruskal-Wallis, Conover's post hoc). $\mathbf{G}-\boldsymbol{I}$, Measurements of thicknesses of the IPL $(\boldsymbol{G})$, length of PKC $\alpha^{+}{ }^{+} \operatorname{BPs}(\boldsymbol{H})$, and the difference between the two $(\boldsymbol{I}) \cdot \mathbf{G}, t_{(4)}=2.689,{ }^{*} p=0.027$ (two-sample $t$ test). $\boldsymbol{H}, t_{(4)}=$ $2.606,{ }^{*} p=0.0298$ (two-sample $t$ test). $I, t_{(4)}=1.072, p=0.172$ (two-sample $t$ test).

tests included a post hoc Conover's test for multiple comparisons of independent samples using a false discovery rate correction. A generalized linear model (GLM) with a Poisson distribution was applied for analysis of connectivity patterns and axon stratification patterns. A Fisher's exact test was used to analyze proportions of ON versus mixed ON/OFF type BPs.

To determine whether parameters were correlated differentially in control versus regenerated retinas, we performed a series of linear regressions for given pairs of parameters. The regression coefficients were calculated for each individual retina (retina used as a blocking factor) for each combination of parameters. The resultant coefficients for correlation (slopes of the linear regressions for each eye), which, if different, would indicate changes in correlative relationships, were then compared for regenerated and control samples and analyzed by Kruskal-Wallis rank-sum tests.

ERG recordings of damaged and regenerated retinas. A custom ERG setup was adapted from that of Makhankov et al. (2004). Recordings were made with anesthetized adult nyx::mYFP zebrafish after $\sim 20 \mathrm{~min}$ of dim light adaptation. After unilateral ouabain injections, damage to retinas was verified by epifluorescence stereomicroscopy; fish that demon- strated complete loss of YFP signal were selected for ERG recordings at 3 DPI or were used for subsequent regeneration studies. All ERG measurements were performed in the early afternoon, from $\sim 12: 00-17: 00$. The light source was a Solis-3C High-Power White LED (Thorlabs). Maximum light intensity was 5000 lux. Light intensity control was achieved using neutral-density filters with defined log unit attenuation. Data were averaged (3-8 responses with a $10 \mathrm{~s}$ ISI), digitized, and analyzed using a computer interfaced PowerLab 200 (AD Instruments). Data were sampled at $1.0 \mathrm{kHz}$ and amplified differentially $1000 \times$ with a band-pass filter between 0.1 and $500 \mathrm{~Hz}$. To quantify the variation of ERG responses between groups, we calculated the sum of the squared deviations among individual control, damaged, and regenerated ERG responses from the grand average of the control ERG responses. The maximum amplitude of each ERG waveform, elicited by a saturating light flash, was scaled to the maximum amplitude of the control grand average and the calculations were made using $\sim 500$ samples per waveform; this analysis permits a comparison of waveform features independent of the amplitude of the b-wave. For analysis of stimulus-response curves, b-wave amplitudes as a function of retinal illuminances were fit with the Naka-Rushton func- 

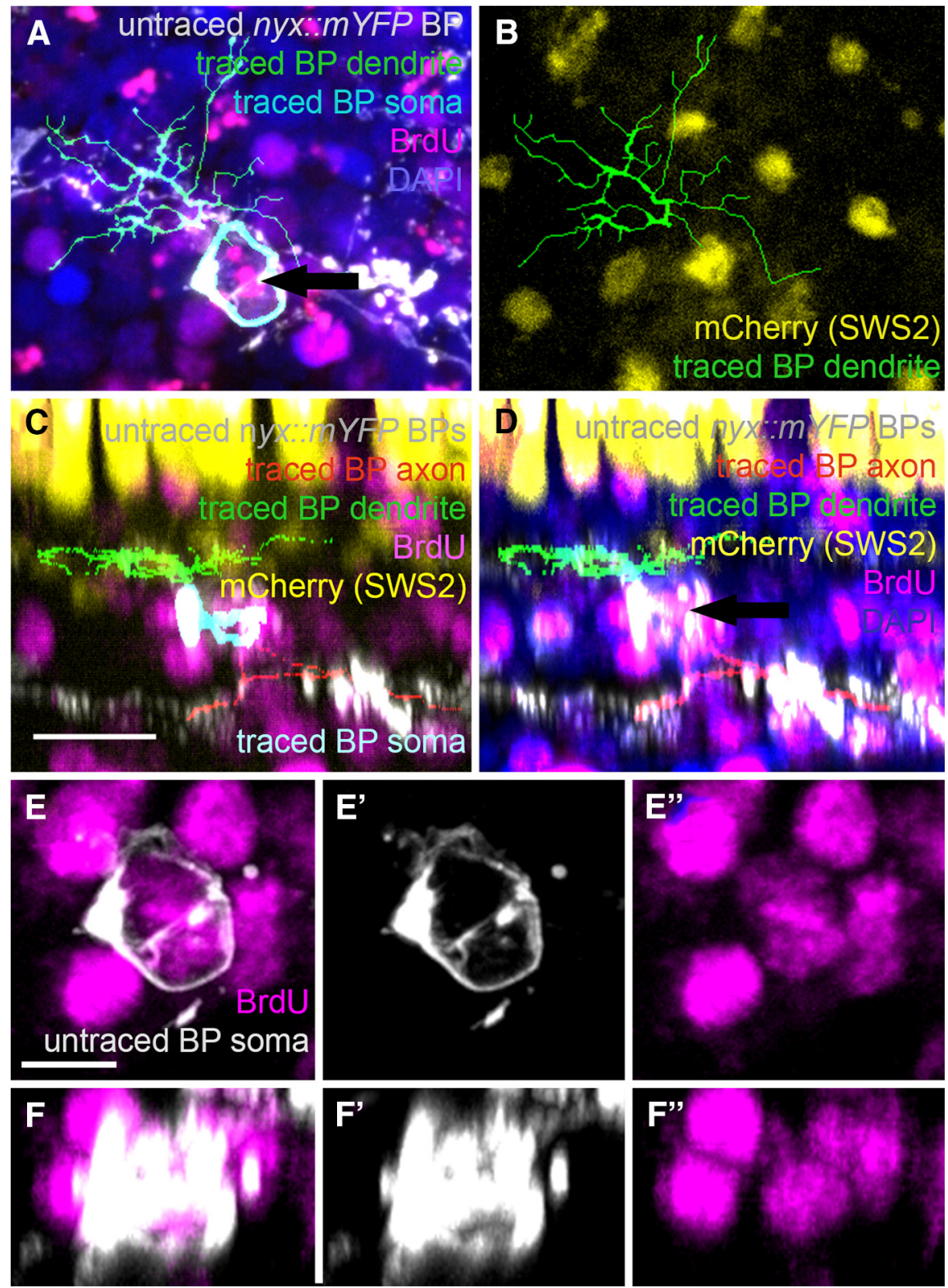

Figure 3. Example of a BrdU ${ }^{+}$, regenerated nyx::mYFP ${ }^{+}$BP. Fish were exposed to BrdU from 4-7 DPI and sampled at $60 \mathrm{DPI}$. $A, X-Y$ projection of an nyx:: $m Y$ FP BP neuron with a BrdU ${ }^{+}$nucleus traced and counterstained with DAPI. B, Same neuron and projection, but soma is not shown and viewed together with synaptic terminals of sws2: $m$ Cherry ${ }^{+}$(blue-sensitive) cones rather than DAPI. C, Same neuron but shown as an $X-Z$ projection traced. $\boldsymbol{D}, X-Z$ projection of the same neuron but soma is untraced and shown with DAPI. $\boldsymbol{E}, \boldsymbol{F}$, Partial projections of this BP neuron's soma in the $X-Y$ orientation $\left(\boldsymbol{E}, \boldsymbol{E}^{\prime}, \boldsymbol{E}^{\prime \prime}\right)$ and $X$-Z orientation $\left(\boldsymbol{F}, \boldsymbol{F}^{\prime}\right.$ $\left.\boldsymbol{F}^{\prime \prime}\right)$ showing z planes corresponding only to the BrdU ${ }^{+}$nucleus surrounded by YFP. Merged $(\boldsymbol{E}, \boldsymbol{F})$ and single-channel images $\left(\boldsymbol{E}^{\prime}\right.$ $\left.\boldsymbol{E}^{\prime \prime}, \boldsymbol{F}^{\prime}, \boldsymbol{F}^{\prime}\right)$ are shown. Arrows in $\boldsymbol{A}$ and $\boldsymbol{D}, \mathrm{DAPI}^{+},{ }^{+}$BrdU ${ }^{+}$nucleus surrounded by YFP. Scale bar in $\boldsymbol{D}$ applies to $\boldsymbol{A}-\boldsymbol{D}$ and is $10 \mu \mathrm{m}$; scale bar in $\boldsymbol{E}$ applies to $\boldsymbol{E}$ and $\boldsymbol{F}$ and is $5 \mu \mathrm{m}$.

tion $\frac{V}{V_{M A X}}=\frac{I^{n}}{I^{n}+K^{n}}$, where $V$ is the b-wave amplitude, $V_{\text {MAX }}$ is the maximum b-wave amplitude, $I$ is illuminance, $K$ is illuminance at halfmaximal amplitude, and $n$ is a coefficient describing the slope of the function.

\section{Results}

Intravitreal injection of $2 \mu \mathrm{M}$ ouabain destroys retinal BPs while preserving cone photoreceptors

To damage the adult zebrafish retina and to create a disrupted environment to challenge regenerated neurons to establish appropriate morphologies and connectomes, we used intravitreal injection of $2 \mu \mathrm{M}$ ouabain. We and others have demonstrated that this lesioning strategy destroys retinal ganglion cells (RGCs), amacrine cells, BPs, and all but a very small number of horizontal cells while sparing photoreceptors and Müller glia (Fimbel et al., 2007; Nagashima et al., 2013; Sherpa et al., 2014). We again confirmed that $2 \mu \mathrm{M}$ ouabain destroyed retinal BPs by observing BP populations that stain with antibodies targeting PKC $\alpha$ and those expressing an $\mathrm{mYFP}$ reporter driven by the nyctalopin promoter nyx::mYFP (Bahadori et al., 2006; Schroeter et al., 2006). In control retinas, these markers identified neurons with cell bodies in the middle to outer half of the INL (Fig. $1 A, D)$ and with axon terminals that stratified either in the inner half of the IPL, consistent with an identity as ON BPs (Connaughton and Nelson, 2000), or in both the inner and outer halves of the IPL, consistent with an identity as mixed ON/ OFF BPs (Connaughton and Nelson, 2000; Fig. $1 A, D, K)$. The $n y x:: m Y F P^{+}$and PKC $\alpha^{+}$BPs were extensively depleted by the ouabain lesion, as observed at 3 DPI in sectioned eyes (Fig. $1 B, E, L$ ) and by microscopic observation of the YFP reporter in eyes of live, anesthetized fish (data not shown). Quantification of $\mathrm{PKC} \alpha{ }^{+}, \mathrm{DAPI}^{+}$, and $n y x:: \mathrm{YFP}^{+}, \mathrm{DAPI}^{+}$cell bodies revealed an $85 \%$ reduction in $\mathrm{PKC} \alpha^{+}$BPs and a $97 \%$ reduction in nyx:: $m Y_{F P}{ }^{+}$BPs at $3 \mathrm{DPI}$ (Figs. $1 G, H, 2 A, B)$. The very few remaining nyx::mYFP ${ }^{+}$cell bodies showed no axonal or dendritic processes (Fig. $1 H$, inset).

Viability of photoreceptors in lesioned retinas was confirmed for all four morphological subtypes of cones using a GFP reporter driven by the sws 1 opsin promoter (UV cones; Takechi et al., 2003; Fig. $1 A, B)$; the antibody ZPR1, which stains cone arrestin3a (Arr3a) in red- and greensensitive double cones (DCs; Renninger et al., 2011; Fig. $1 A, B, D, E)$; and an mCherry reporter driven by the sws 2 opsin promoter (blue cones; Salbreux et al., 2012; Fig. $1 D, E)$. Cones retained their characteristic morphologies at the 3 DPI sampling time (Fig. $1 B, E$ ), similar to the findings after selective ablation of $x f z 43$ BPs in larval zebrafish (D'Orazi et al., 2016), but remarkable given the widespread destruction of inner retinal neurons in the present study. The loss of BPs throughout the retina, but the preservation of cones, was observed consistently in lesioned samples sectioned at 3 DPI (Fig. 1H) or observed live by fluorescence stereomicroscopy (data not shown), confirming $2 \mu \mathrm{M}$ ouabain as a reliable method to destroy BPs (Fimbel et al., 2007; Nagashima et al., 2013; Sherpa et al., 2014).

Regeneration of retinal BPs after intravitreal injection of $2 \mu \mathrm{M}$ ouabain

To analyze the morphologies and connectivities of regenerated BPs, we selected 60 DPI as the time of analysis because we have demonstrated previously that, in addition to histological regen- 
A

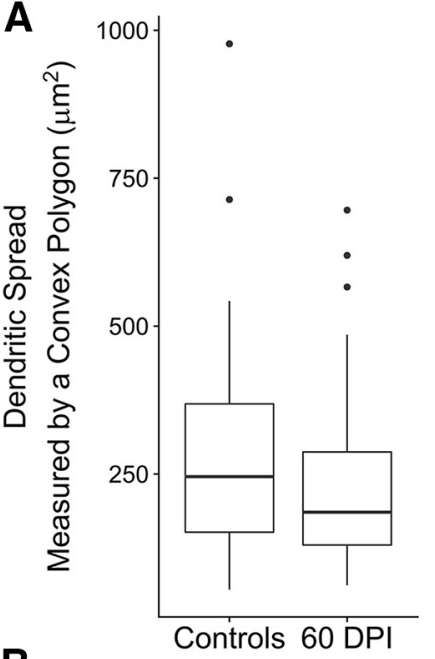

B

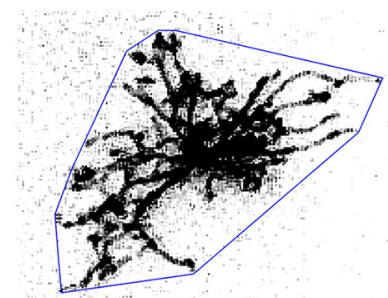

Control-Polygon

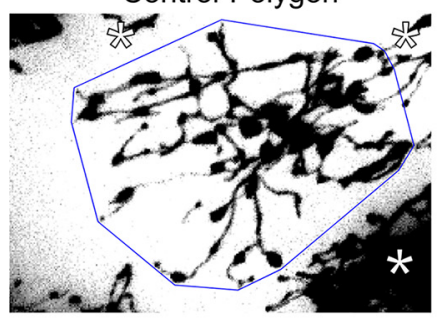

60 DPI-Polygon

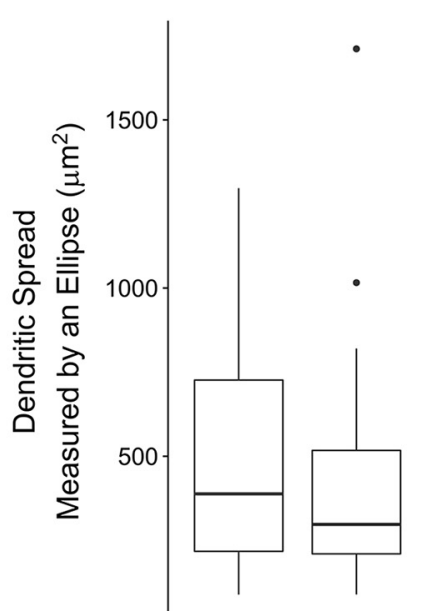

C

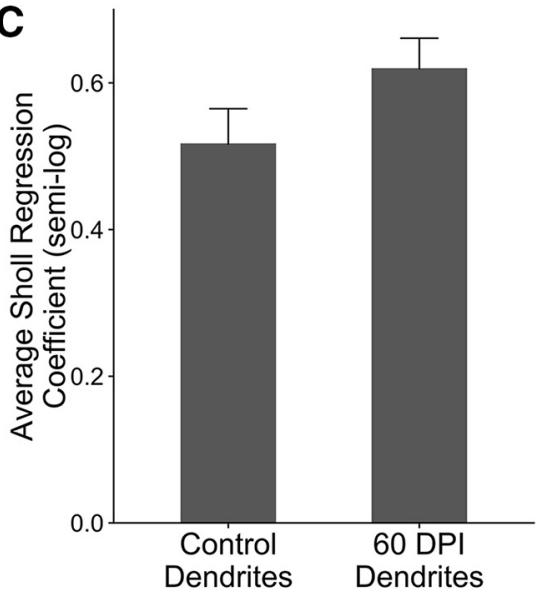

Controls $60 \mathrm{DPI}$

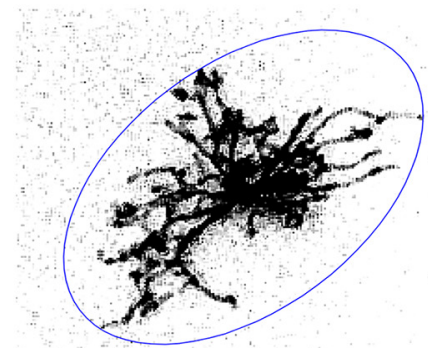

Control-Ellipse

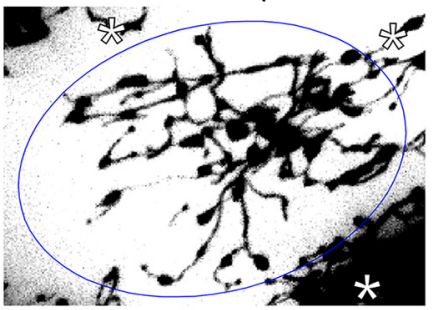

60 DPI-Ellipse

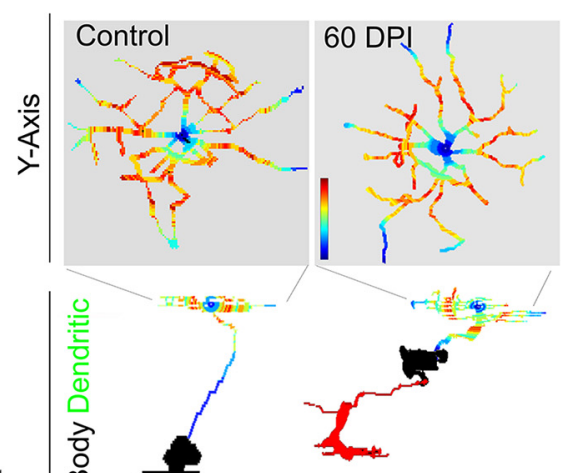

S1S3S4 Tristratified

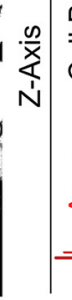

文

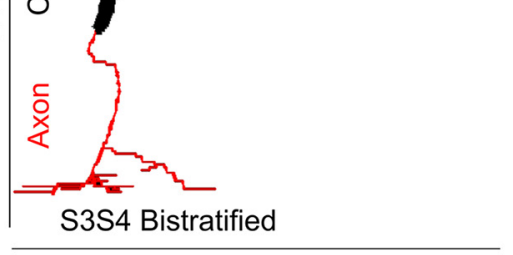

Figure 4. Dendritic field sizes and complexities of nyx::mYFP BPs are restored in regenerated retina. $A$, Boxplots showing distribution of dendritic field sizes as measured by convex polygons or by ellipses. In the boxplots, the band inside the box represents the median, the top and bottom of the box represent the $25^{\text {th }}$ and $75^{\text {th }}$ percentiles, the whiskers represent the 1.5 interquartile range, and the filled circles represent outliers. $B$, Convex polygons and ellipses (blue) drawn around the dendritic fields of examples of control and regenerated BPs. $C$, Sholl analysis of complexity of dendritic trees of control and regenerated nyx::mYFP BPs. Top, Mean ( \pm SEM) Sholl (semi-log) regression coefficients for BPs in control and regenerated retinas. Bottom: $X-Z$ views of selected, traced BPs. Top ( $y$-axis) view of dendritic trees are color coded by heat maps to demonstrate relative numbers of dendrite branches that cross circles of increasing distance from the initial branch point. Hotter colors represent more dendrites and cooler colors represent fewer dendrites. *Dendrites of neighboring nyx::mYFP BPS.

eration, simple, visually mediated reflexes and behaviors are restored by 60 DPI (Sherpa et al., 2014). Quantification of regenerated, $\mathrm{PKC} \alpha^{+}$, $\mathrm{DAPI}^{+}$, or $n y x:: m Y F P^{+}$, $\mathrm{DAPI}^{+}$BPs showed partial restoration of BP numbers at $60 \mathrm{DPI}$, with more robust restoration of PKC $\alpha^{+}$BPs than of nyx::mYFP ${ }^{+}$BPs (Fig. $2 A, B$ ). Furthermore, restoration of nyx:: $m Y F P^{+}$BPs was widely variable, with some retinas showing substantial regeneration of these neurons, but others showing very little, resulting in large SEMs of neuron numbers at 60 DPI (Fig. 2B). Percentage recovery of PKC $\alpha^{+}$BPs and $n y x:: m Y F P^{+}$BPS were $33.18 \pm 9.2($ SEM $) \%$ and $12.86 \pm 10.7 \%$, respectively. Numbers of $\mathrm{HuC} / \mathrm{D}^{+}$amacrine cells and RGCs are also not fully restored by $60 \mathrm{DPI}$ after this type of ouabain lesion (Sherpa et al., 2014).

The nyx:: $m$ YFP BPs have been considered to be a subset of the PKC $\alpha$ BPs in larval zebrafish retina (Schroeter et al., 2006). However, analysis of $\mathrm{PKC} \alpha$, nyx:: $m Y F P$ colabeling in adult retina revealed virtually no overlap in these two populations (Figs. $1 \mathrm{~K}$, $2 C-E$ ), suggesting differences in BPs of adult versus larval retina.
Table 1. Sholl analysis of dendrite branching patterns of nyx::mYFP bipolar neurons in control and regenerated retinas

\begin{tabular}{llrl}
\hline Parameter & $\begin{array}{l}p \text {-value (control } \\
\text { vs } 60 \mathrm{DPI})\end{array}$ & \multicolumn{1}{c}{$\chi^{2}$ ort } & $\begin{array}{l}\text { Statistical } \\
\text { test }^{a}\end{array}$ \\
\hline Maximum no. of intersections & 0.345 & $\chi_{(1)}^{2}=0.886$ & $\mathrm{~A}$ \\
Sum of intersections & 0.4817 & $\chi_{(1)}^{2}=0.016$ & $\mathrm{~A}$ \\
Radius at maximum no. of intersections & 0.900 & $\chi_{(1)}^{2}=0.495$ & $\mathrm{~A}$ \\
Critical-value & 0.8741 & $t_{(47.0)}=-0.159$ & $\mathrm{~B}$ \\
Radius of critical-value & 0.472 & $\chi_{(1)}^{2}=0.517$ & $\mathrm{~A}$ \\
Enclosing radius & 0.108 & $t_{(54.77)}=1.632$ & $\mathrm{~B}$ \\
Kurtosis of the sampled data & 0.870 & $\chi_{(1)}^{2}=0.027$ & $\mathrm{~A}$ \\
Kurtosis of the fitted data & 0.736 & $t_{(47.9)}=0.339$ & $\mathrm{~B}$ \\
Skewness of the sampled data & 0.8451 & $t_{(49.4)}=-0.196$ & $\mathrm{~B}$ \\
Skewness of the fitted data & 0.775 & $t_{(47.22)}=-0.287$ & $\mathrm{~B}$ \\
\hline
\end{tabular}

${ }^{a} \mathrm{~A}$, Kruskal-Wallis; B, Welch's two-sample $t$ test. 

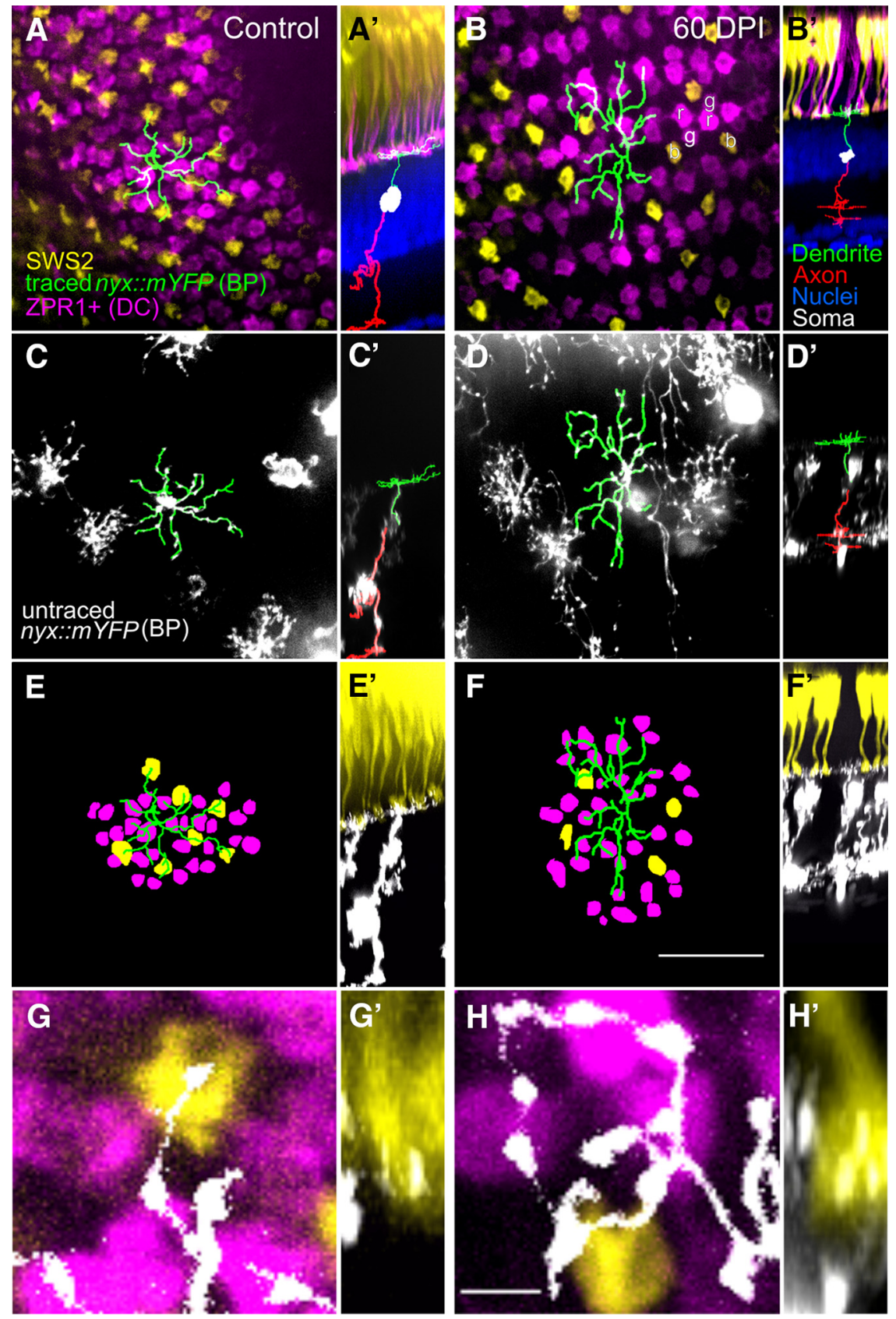

Figure 5. Determination of cone connectivity patterns of nyx::mYFP BPs in control and regenerated retinas. $\boldsymbol{A}, \boldsymbol{B}$, Traced individual BP dendritic fields overlaid with view of synaptic terminals of blue-sensitive (SWS2; b in $\boldsymbol{B}$ ) and ZPR1 ${ }^{+}$DCs in control $(\boldsymbol{A})$ and regenerated $(\boldsymbol{B})$ retinas. Position in cone mosaic and more intense labeling of red-sensitive cones (LWS; $r$ in $\boldsymbol{B}$ ) permits their identification as distinct from the green-sensitive cones (RH2; $g$ in $\boldsymbol{B}$ ). $\boldsymbol{C}, \mathbf{D}$, Traced individual BP dendritic fields overlaid by the microscopic image showing examples of untraced $B P$ s in control $(\boldsymbol{C})$ and regenerated $(\boldsymbol{D})$ retinas. $\boldsymbol{E}, \boldsymbol{F}$, Traced individual BP dendritic fields overlaid with traced cone terminals in control $(\boldsymbol{E})$ and regenerated $(\boldsymbol{F})$ retinas. $\boldsymbol{G}, \boldsymbol{H}$, Higher-magnification Z-projections showing dendritic tips associated with cone terminals. $\boldsymbol{A}^{\prime}-\boldsymbol{H}^{\prime}$ depict partial projections from image stacks to show resliced radial view of analyzed BPs. Scale bar in $\boldsymbol{F}$ applies to $\boldsymbol{A}-\boldsymbol{F}$ and is $20 \mu \mathrm{m}$; scale bar in $\boldsymbol{H}$ applies to $\boldsymbol{G}$ and $\boldsymbol{H}$ and is $2.5 \mu \mathrm{m}$.

No colabeled BPs were detected at 3 DPI and the numbers of colabeled BPs observed at 60 DPI were not significantly different from controls (Figs. $1 L, M, 2 C-E$ ), suggesting that both populations were restored, albeit in reduced numbers (and widely variable for $n y x:: m Y F P$ BPs). However, ratios of $\mathrm{PKC} \alpha^{+}$to $n y x:$ : $m \mathrm{YFP}^{+}$BPs were not restored to control ratios (Fig. $2 F$ ), likely reflecting the more substantial numbers of regenerated $\mathrm{PKC} \alpha$ BPs. We and others have observed additional histological abnormalities in regenerated retina, including ectopic cell bodies
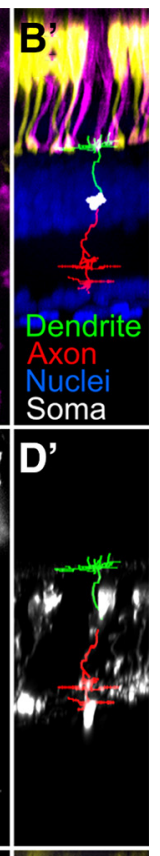

within plexiform layers (laminar fusions, Hitchcock et al., 1992; Sherpa et al., 2014), the production of supernumerary neurons (Sherpa et al., 2014; Powell et al., 2016), and/or neuronal cell types that were not the types injured by the initial damage (Powell et al., 2016). In the present study, IPL layer thickness was significantly reduced at 60 DPI compared with controls (Fig. 2G) and the total length of $\mathrm{PKC} \alpha^{+}$BPs was also reduced at 60 DPI (Fig. $2 H$ ). Interestingly, there were no differences in the length of the BP neurons when the IPL was subtracted from the measurements (Fig. 2I), indicating that the primary histological abnormalities may reside within the IPL. Some regenerated $\mathrm{PKC} \alpha{ }^{+}$BPs showed apparently unusual axon morphologies (Fig. 1C) and we occasionally observed nyx::mYFP ${ }^{+}$cell bodies displaced in the GCL (Fig. $1 M$, inset). Therefore, we performed detailed morphometric analyses on a subset of $\mathrm{BPs}$ to test the hypothesis that regenerated BPs restored morphologies and connectivities similar to those of their undamaged counterparts despite these histological disruptions.

\section{Complexity of dendritic trees of}

regenerated $n y x:: m Y F P$ BPs is restored

To analyze dendritic tree morphologies of BPs, we took advantage of the variegated expression of the mYFP reporter in the adult nyx::mYFP transgenic zebrafish (Schroeter et al., 2006; Saade et al., 2013) because this permitted the visualization of individual BPs in retinal whole mounts by confocal microscopy. In addition, this BP population was most reliably destroyed by the $2 \mu \mathrm{M}$ ouabain lesion and returned in reduced numbers, facilitating the analysis of individual neurons (Fig. 2A,B). We verified further that $n y x:: m Y F P^{+}$BPs were truly regenerated at 60 DPI (and did not simply survive the lesion) using BrdU incorporation studies. BrdU immersion from 4-7 DPI or from 7-12 DPI, followed by sampling at $60 \mathrm{DPI}$, each revealed numerous $\mathrm{BrdU}^{+}$, nyx::mYFP ${ }^{+} \mathrm{BPs}$, suggesting that BPs are generated over a protracted time frame and demonstrating true regeneration of these BPs (example in Fig. 3). The BrdU ${ }^{+}$, nyx::mYFP ${ }^{+}$BPs displayed dendritic and axonal processes (Fig. $3 A-D$ ) and apparent dendritic tip contacts with sws $2: m$ Cherry $^{+}$cones (Fig. $3 B-D$ ). For the morphometric analyses, six $\mathrm{BrdU}^{+}$regenerated BPs from these experiments were included, along with 26 regenerated BPs from experiments that did not include a BrdU immersion step.

The dendritic area of traced BPs was measured by drawing a convex polygon around the perimeter of the dendritic tree (Kraft et al., 2006) and separately by fitting an ellipse around the perimeter of the dendritic tree (Li et al., 2012; Fig. 4A,B). These mea- 
surements revealed median dendritic spreads of 200-250 $\mu \mathrm{m}^{2}$ (Fig. $4 A$, polygon) and $350-400 \mu \mathrm{m}^{2}$ (Fig. $4 A$, ellipse) for both sample populations. Neither measures detected a significant difference in spreads of regenerated BP dendritic fields $(n=33)$ compared with undamaged control BPs $\left(n=26 ; \chi_{(1)}^{2}=1.015\right.$, $p=0.314$ for polygon; $\chi_{(1)}^{2}=1.078, p=0.300$ for ellipse; Kruskal-Wallis rank-sum test; Fig. $4 A$ ). Control and 60 DPI dendritic spreads showed similar Feret's diameters $\left(\chi_{(1)}^{2}=0.525, p=\right.$ $0.525 ; n=26$ and 33; Kruskal-Wallis) with median diameters of $23.9 \mu \mathrm{m}$ and $25.0 \mu \mathrm{m}$, respectively. Control and 60 DPI dendritic spreads also were similar in roundness $\left(t_{(45.5)}=-0.494, p=\right.$ $0.623 ; n=26$ and 33; Welch's two-sided $t$ test) with mean values \pm SEM of $0.738 \pm 0.037$ and $0.715 \pm 0.025$, respectively. One regenerated BP displayed two primary dendrites supporting two separate dendritic trees (and will be shown later in the Results). However, each of these trees showed dendritic spreads and Feret's diameters within the ranges described above.

Sholl analysis (Sholl, 1953) was used as an additional measure of morphology and complexity of dendritic trees of identified $n y x:: m Y F P$ BPs. Sholl analysis measures the number of dendritic branches that intersect a series of concentric circles drawn at increasing distance from a defined center point of the dendritic network (Fig. 4C). Outputs of Sholl analysis provide quantitative information regarding the density of branches, distributions of branches, and branching patterns of the dendritic trees of identified neurons. This analysis revealed no differences in the Sholl regression coefficients (Fig. $4 C ; \chi_{(1)}^{2}=3.067, p=0.08$ ) and $Y$ intercepts (data not shown; $\chi_{(1)}^{2}=2.644, p=0.104$ ) using the semi-log method, in regenerated nyx::mYFP BP dendritic trees compared with control trees (Kruskal-Wallis rank-sum tests; $n=$ 26 for controls; $n=32$ for 60 DPI). In addition, output of Sholl analysis using the linear method also showed no significant differences in regenerated nyx::mYFP BP dendritic tree morphologies compared with controls (Table 1).

\section{Cone connectivity patterns of regenerated nyx::mYFP BPs are restored}

To determine the cone connectivity patterns of BPs, identified $n y x:: m Y F P$ BPs were visualized in retinal whole mounts together with blue-sensitive cones (sws2:mCherry) and ZPR1-stained DCs (Fig. $5 A, B$ ). Several samples permitted further discrimination of the red- vs green-sensitive (LWS vs RH2 opsin-expressing) members of each DC pair, because the ZPR1 antibody more strongly labeled the red-sensitive (LWS) member (Fig. 5A,B). Furthermore, the organization of the cone mosaic in adult zebrafish retinas permits the assignment of red versus green in regions where clearly aligned rows of DCs display alternating orientations (Allison et al., 2010; Li et al., 2012). Connectivity assignments were made using image stacks that included traced (Fig. $5 A-F$ ) and untraced (Fig. 5G,H) nyx::mYFP BPs, supervised assignment of cone terminal identity (Fig. $5 E, F$ ), and verification of dendritic tip (end point) association with cone terminals (Fig. $5 G, H)$. Assignments and assessments of cone-BP synapses were in many cases further verified in $X-Z$ projections (Fig. $5 A^{\prime}-H^{\prime}$; see also Fig. $3 D$ ). The majority of regenerated nyx::mYFP BPs made connections to all of the identified cone subtypes, similar to the range of connections made by undamaged control nyx::mYFP BPs (Table 2).

The numbers of dendritic tips of each identified nyx::mYFP BP dendritic tree were not significantly different in regenerated $(\overline{\mathrm{x}}=$ $17.55, \mathrm{SD}=6.07, n=29)$ versus control retinas $(\overline{\mathrm{x}}=15.8, \mathrm{SD}=$ 6.03, $n=16$; $t_{(31.22)}=0.923, p=0.363$; Welch's two-sample $t$ test; Fig. $6 A$ ). The total number of end points per dendritic tree
Table 2. Connectivity patterns of nyx::mYFP bipolar neurons observed in control and regenerated retinas

\begin{tabular}{lll}
\hline & No. of neurons & \\
\cline { 2 - 3 } Connectivities $^{a}$ & Control & 60 DPI \\
\hline BGRX & 10 & 6 \\
BGX & 2 & 0 \\
BRX & 0 & 2 \\
GRX & 1 & 2 \\
GX & 0 & 1 \\
\hline
\end{tabular}

${ }^{a}$ Connections observed to SWS2 (B), RH2 (G), and LWS (R) cones, as well as unassigned (X) connections.

ranged from 7 to 30, similar to ranges observed previously (Li et al., 2012). The regenerated BP with two dendritic trees displayed a total of 42 end points.

Cone connectivity patterns were analyzed further to test whether the number of connections regenerated nyx::mYFP BPs made to each type of cone were similar to or different from those of control nyx::mYFP BPs. Unassigned connections were most likely connections to UV cones or rods (Li et al., 2012), but we cannot rule out the possibility of end points lacking connections. Figure 6 plots the distributions of assigned cone-specific connections, as well as unassigned/presumed UV or rod connections. We used a GLM to determine whether connectivity patterns were different in control versus regenerated retina (i.e., do nyx::mYFP $\mathrm{BPs}$ in regenerated retina connect more or less frequently to any specific cone subtype than in control retina?). The strength of the GLM is that it provides a framework to allow comparisons of two or more variables without assuming normal distributions. Here, we used a Poisson regression type GLM to compare numbers of photoreceptor-specific contacts for control versus regenerated nyx::mYFP BPs. The distributions of photoreceptor-specific connections were found to be statistically similar for control and regenerated BPs $(z=$ $-0.75, p=0.453)$. Therefore, the regenerated nyx::mYFP BPs developed dendritic trees that received input from similar combinations of cones (Table 2) and displayed similar numbers of connections to each cone subtype (Fig. 6).

\section{Synaptic connections to cones are also restored in regenerated PKC $\alpha^{+}$BPs}

To further test for the restoration of cone-BP synapses in retina that regenerated after destruction of inner retinal neurons, we visualized PKC $\alpha^{+}$BPs in sectioned retinas. The PKC $\alpha^{+}$BP population is too dense to visualize as individual neurons in whole mounts, but offers versatility for multicolor confocal imaging in sectioned retinas together with UV-sensitive (SWS1) cones that are $\mathrm{GFP}^{+}$. We also verified that a synapse-specific marker, SV2 (Yazulla and Studholme, 2001) was present and properly localized in regenerated adult retina. In undamaged control retinas, SV2 was localized to the OPL and IPL (Fig. 7A) and UV-sensitive (sws1:GFP) cones made apparent synaptic contacts with $\mathrm{PKC} \alpha^{+}$ BPs (Fig. $7 A, D, G$ ). At 3 DPI, an SV2 ${ }^{+}$IPL was not detectable, whereas the OPL was still stained by SV2 (Fig. $7 B$ ). Interestingly, SV2 staining in the 3 DPI photoreceptor layer was more broadly distributed and appeared to stain some photoreceptor axons and axon terminals (Fig. $7 B$ and inset). This finding indicates that, despite their apparently normal morphology, zebrafish cone photoreceptors may change at the subcellular level in response to injuries that destroy their synaptic partners. To our knowledge, this is the first reported indication of such a change. However, these cone terminals still displayed synaptic clefts (Fig. 7 E, H). At $60 \mathrm{DPI}$, the SV2 staining pattern was largely restored, supporting 
A

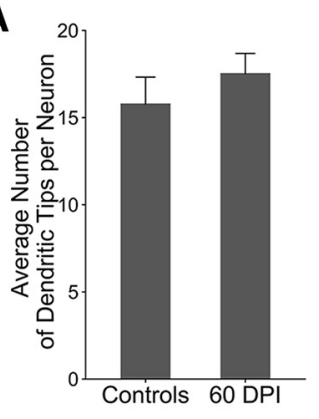

B

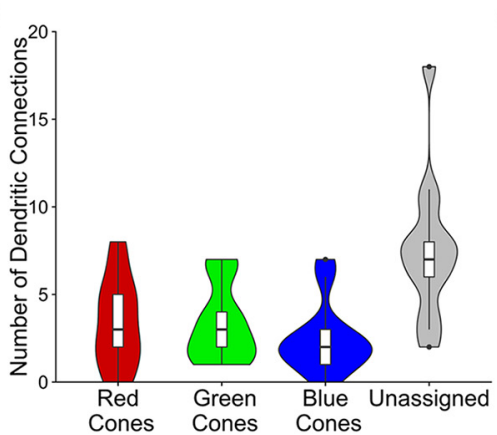

C

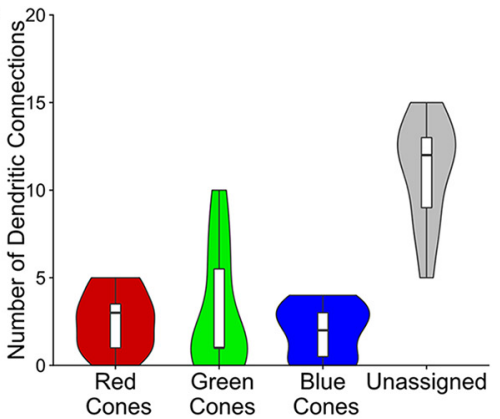

Figure 6. Re-establishment of BP dendritic connections with photoreceptors. $\boldsymbol{A}$, Column graph showing average number of dendritic tips per nyX:: $m Y F P$ dendritic tree in control and regenerated retinas. $\boldsymbol{B}, \boldsymbol{C}$, Distributions of dendritic connections to identified and unassigned photoreceptor subtypes for nyx:: $m Y F P$ BPs in control $(\boldsymbol{B})$ and regenerated $(\boldsymbol{C})$ retinas. Shapes of violin plots were obtained by using a kernel density estimator to generate a smoothened histogram, mirrored along the $x$-axis, and then rotated. The width of each plot was determined by the proportion of BPs making a given number of connections to that photoreceptor subtype at that point. In the boxplots within, the horizontal line inside each box represents the median, the top and bottom of the box represent the $25^{\text {th }}$ and $75^{\text {th }}$ percentiles, the whiskers represent the 1.5 interquartile range, and the filled circles represent outliers.
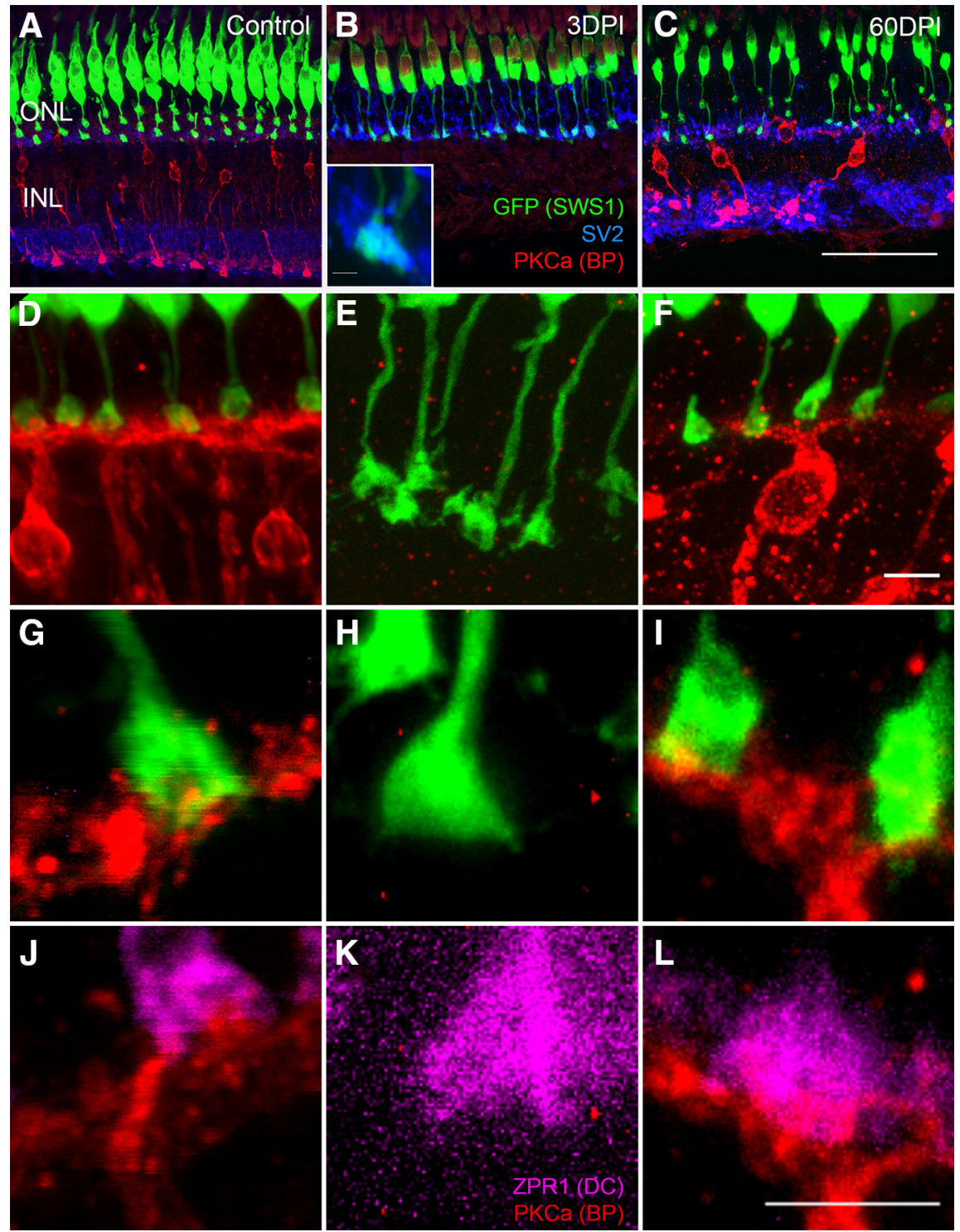

Figure 7. Restoration of synapses between cone photoreceptors and $\mathrm{PKC} \alpha{ }^{+}$BPs in regenerated retina. $\boldsymbol{A}-\boldsymbol{C}$, Distribution of presynaptic marker SV2 in undamaged retinas $(\boldsymbol{A})$ and in damaged retinas at $3 \mathrm{DPI}(\boldsymbol{B})$ and $60 \mathrm{DPI}(\boldsymbol{C})$. Inset in $\boldsymbol{B}$ shows SV2 immunofluorescence associated with a UV-GFP terminal. $\boldsymbol{D}-\boldsymbol{F}, \mathrm{PKC} \alpha{ }^{+}$BPs are absent at $3 \mathrm{DPI}(\boldsymbol{E})$ but regenerated with connections to GFP ${ }^{+}$UV (SWS1) cones at $60 \mathrm{DPI}(\boldsymbol{F}) . \mathbf{G}-\mathbf{I}$, Higher-magnification views of UV cone synaptic terminals in control (G), 3 DPI $(\boldsymbol{H})$, and $60 \mathrm{DPI}(\boldsymbol{I})$ retinas, showing loss and restoration of synapses with PKC $\alpha{ }^{+}$BPs. $\boldsymbol{J}-\boldsymbol{L}$, High-magnification views of ZPR1 DC synaptic terminals. Scale bar in $\boldsymbol{C}$ applies to $\boldsymbol{A}-\boldsymbol{C}$; scale bar in $\boldsymbol{F}$ applies to $\boldsymbol{D}-\boldsymbol{F}$; and scale bar in $\boldsymbol{L}$ applies to $\mathbf{G}-\boldsymbol{L}$ and all are $5 \mu \mathrm{m}$. the hypothesis that the regenerated cone-BP synapses are likely functional, although some gaps in the IPL were present and occasional labeling of structures beyond the OPL was observed (Fig. 7C). At 60 DPI, UV cone contacts with PKC $\alpha^{+}$ BPs were observed (Fig. $7 F, I$ ). Cone-BP contacts between ZRP1 ${ }^{+}$DCs and PKC $\alpha$ BPs were also observed to be present in control retinas, lost at 3 DPI, and then restored at $60 \mathrm{DPI}$ (Fig. $7 J-L$ ).

\section{Axons of regenerated nyx:: $m Y F P$ BPs display a diversity of stratification patterns and complexities}

We next tested whether axons of regenerated BPs displayed the same diversity of stratification patterns in the IPL as their undamaged counterparts. This was possible using the $X-Z$ projections of identified nyx::mYFP BPs in imaged whole-mounted retinas for 20 of the traced, regenerated BPs (examples shown in Fig. $8 A, B$ ). $\mathrm{BP}$ neurons of the zebrafish can show a variety of stratification patterns, each likely indicative of function as an ON BP (synapsing in deep layers, S4-S6), an OFF BP (synapsing closer to the INL, S1-S3), or a mixed ON/OFF BP (synapsing in a combination of these layers) (Connaughton and Nelson, 2000; Connaughton et al., 2004). The stratification patterns of nyx:: $m Y F P$ BPs in control retinas revealed some diversity, with individual neurons showing distinct stratification combinations. Analyzed BPs showed mono-stratified, di-stratified, and tri-stratified axon morphologies (Table 3). The majority could be tentatively assigned to a function as a mixed ON/OFF BP. Although the IPL of regenerated retinas showed reduced thickness, regenerated nyx::mYFP BPs also displayed a diversity of apparent stratification patterns that could tentatively be designated 


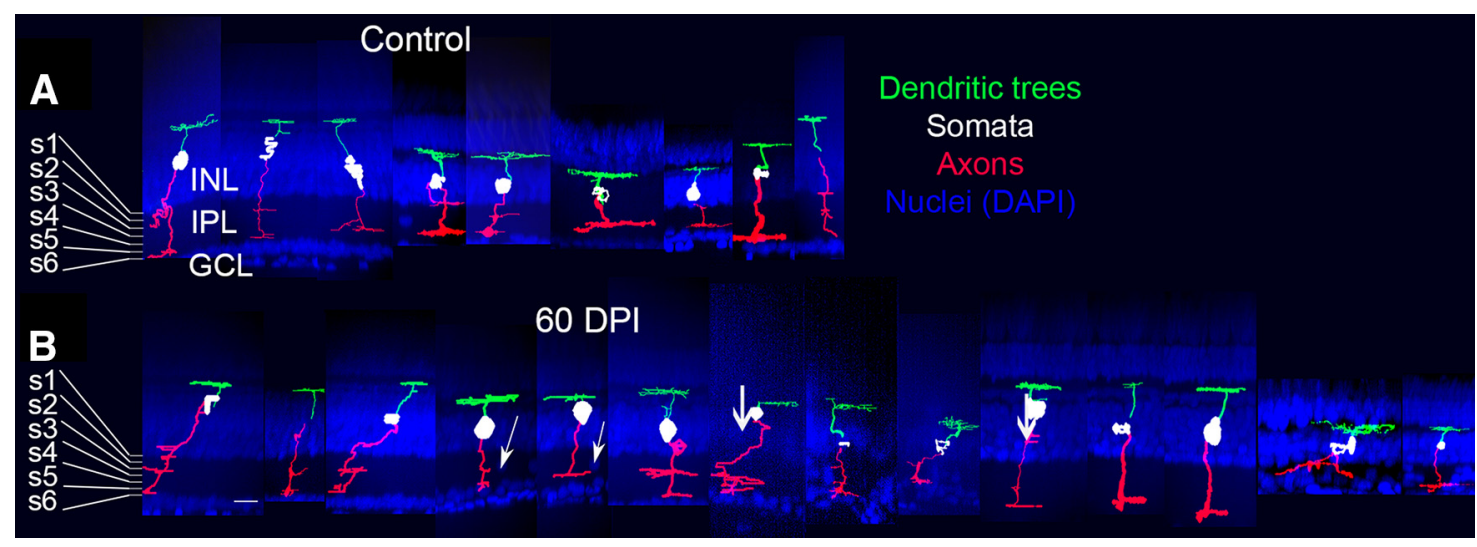

Figure 8. Stratification patterns of nyx::mYFPBPs are restored in regenerated retina. $A, B, X-Z$ reconstructions of traced nyx::mYFPBPs. BPs were traced, colorized, and image stacks were resliced with the nuclear marker DAPI to show retinal laminae in control $(\boldsymbol{A})$ and regenerated $(\boldsymbol{B})$ retinas. The IPL was divided into six equal layers or strata. s1-s6, Strata of IPL. Arrows in $\boldsymbol{B}$ show selected examples of "laminar fusions" (nuclei in IPL) seen regularly in regenerated retinas. The last two neurons in $\boldsymbol{B}$ were derived from a BrdU incorporation experiment and were BrdU ${ }^{+}$.

Table 3. Axon stratification patterns of nyx::mYFP bipolar neurons in control and regenerated retinas

\begin{tabular}{|c|c|c|c|c|c|}
\hline \multirow[b]{2}{*}{ Stratification layers } & \multicolumn{2}{|c|}{ No. of Neurons } & \multirow[b]{2}{*}{ Predicted function } & \multicolumn{2}{|c|}{ No. of Neurons } \\
\hline & Control & $60 \mathrm{DPI}$ & & Control & $60 \mathrm{DPI}$ \\
\hline S4S5 & & 3 & & & \\
\hline S5 & 1 & 3 & & & \\
\hline 5556 & 1 & 1 & ON & $3(27 \%)$ & $9(45 \%$ \\
\hline S6 & 1 & 1 & & & \\
\hline 545556 & & 1 & & & \\
\hline S156 & 1 & 1 & & & \\
\hline 515356 & 1 & 2 & & & \\
\hline S2S4 & & 3 & & & \\
\hline S2S5 & 1 & 1 & Mived ON/OFF & $8(73 \%)$ & $11,55 \%$ \\
\hline$S 256$ & 2 & & IVIXea Uiv/UrF & & \\
\hline S3S4 & 1 & & & & \\
\hline S3S5 & 1 & & & & \\
\hline 5356 & 1 & 4 & & & \\
\hline
\end{tabular}

with functional assignments as mixed ON/OFF BPs (Table 3). The regenerated nyx::mYFP BPs did include some distinct patterns not seen in the sample set derived from control retinas (Table 3). However, statistical analysis revealed that the number of stratifications of regenerated BPs matched those of control retinas (GLM; $z=-0.084, p=0.933$ ) and the types of stratifications (ON vs mixed ON/OFF) were also similar (Table 3; odds ratio $=0.6186, p=0.702$; Fisher's exact test). We conclude that more than a single type of stratification pattern were restored in the regenerated nyx::mYFP BPs and that highly abnormal stratification patterns did not emerge.

Sholl analysis (Sholl, 1953) using the log-log method found no differences in the Sholl regression coefficient of axons from control $(\overline{\mathrm{x}}=2.88, \mathrm{SD}=0.404, n=12)$ versus regenerated nyx:: $m$ YFP BPS $\left(\overline{\mathrm{x}}=3.03, \mathrm{SD}=0.324, n=23 ; t_{(18.95)}=1.135, p=\right.$ 0.271 ; Welch's two-sided $t$ test; Fig. 9). In addition to using the $\log -\log$ method, axons were also analyzed using the linear method (Ferreira et al., 2014). Of the output metrics measured, only the maximum number of intersections was significantly reduced in regenerated BPs compared with control BPs (Table 4). Because there was no difference in the total number of intersections, the size of the overall area occupied by the axon, or the distribution of the axon branches, this finding suggests that axon branches of regenerated BPs are more evenly distributed along the length of the axon (its enclosing "Sholl sphere") than those of control retinas.

\section{Combinations of morphologies of nyx::mYFP BPs are restored}

When dendritic spread, connectivity, and stratification data are considered together, the nyx::mYFP ${ }^{+}$BPs appeared to constitute a heterogeneous population that most closely corresponded with the RGBUV and/or RGBRod (ON or MIXED) BPs documented by Li et al. (2012) and the Group b (ON type) and Group a/b (MIXED) type BPs documented by Connaughton et al. (2004). We therefore next tested whether combinations of dendritic or axon parameters displayed distinct covariances in regenerated versus control BP populations, which would suggest differential biases in the production of $n y x:: m Y F P$ BPs with specific functional attributes. We found that dendritic spread was not differentially correlated with dendritic complexity (Sholl regression coefficient), dendritic spread was not differentially correlated with axon complexity, dendritic complexity was not differentially correlated with axon complexity, and the number of dendritic end points was not differentially correlated with axon complexity in control or regenerated BPs (Fig. $10 A-C$ ). Several additional potential correlations were evaluated using a blocking factor in case of biases related to individual eyes and these also revealed no correlations and no significant differences in control versus regenerated BPs (Table 5). Visualization of three distinct morphological measurements, dendritic spread, total number of end points, and the Sholl regression coefficient for axons (log-log), using 3D scatterplots also did not reveal any differential clustering in regenerated versus control BPs (Fig. 10D). Finally, dendritic spreads were not significantly different in the $O N$ versus mixed ON/OFF subsets of nyx::mYFP BPs and this was true for both control and regenerated BPs (Fig. 10E). Therefore, regenerated BPs showed no biases favoring particular combinations of morphologies and did not demonstrate new correlations that were not observed in undamaged BPs.

Because our morphometric analyses did not reveal substantial, statistically significant differences in sizes or complexities of dendritic trees or axons or in connectivities of BPs in the two experimental conditions, we also assessed BP morphologies qualitatively. Projected surface renderings are shown in Figure 11 from two different viewpoints. Our conclusion from qualitative inspection of these projections is that the regenerated BPs show a range of morphologies that are largely similar to the range of morphologies of control BPs, suggesting a lack of bias in the production of any one morphology during retinal regeneration. The notable exceptions include the one regenerated BP display- 

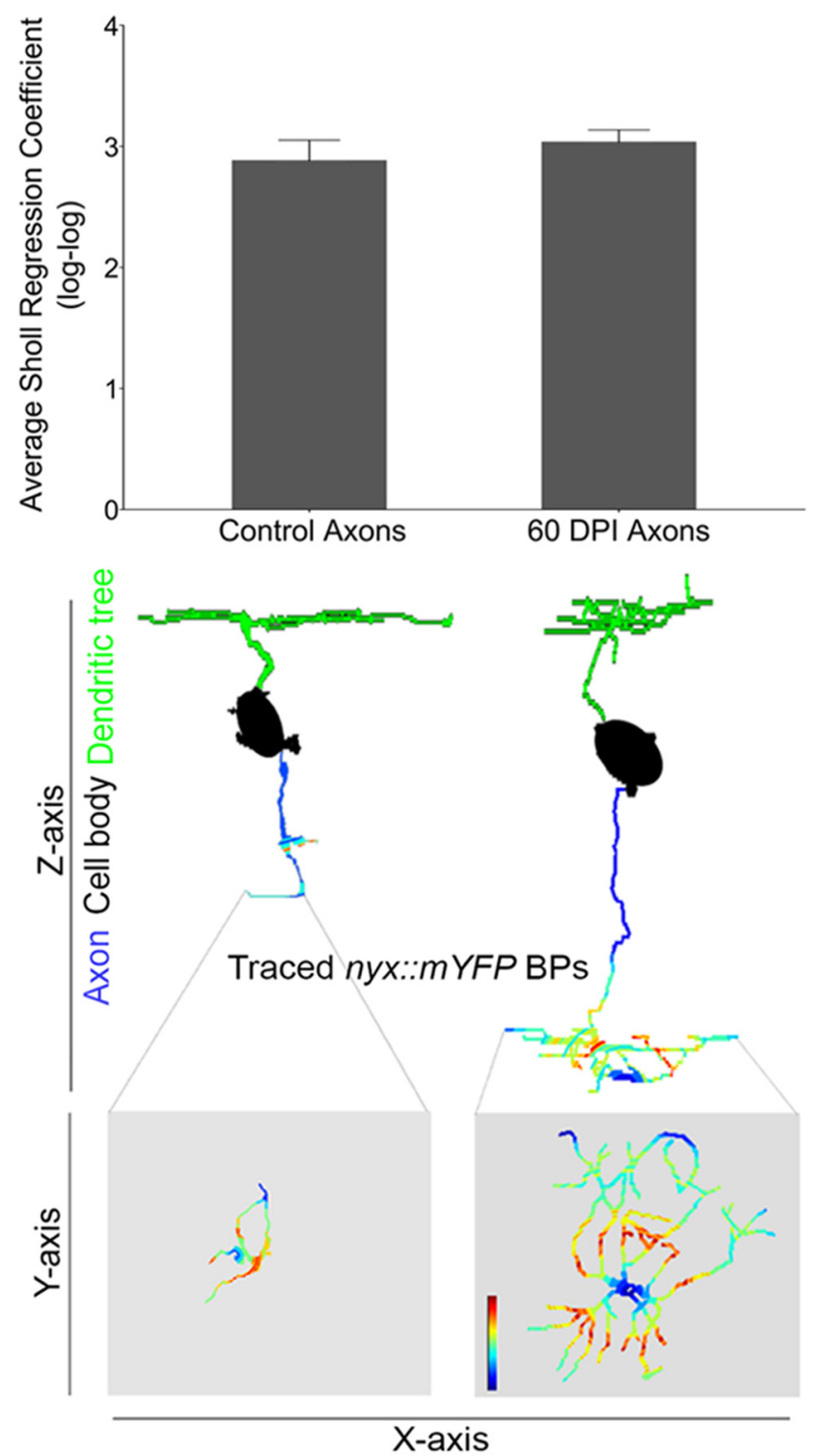

Figure 9. Axon terminal complexities of nyx::mYFP BPs are restored in regenerated retina. Sholl analysis of complexity of dendritic trees of control and regenerated nyx::mYFP BPs. Top, Mean ( \pm SEM) Sholl $(\log -\log )$ regression coefficients for BPs in control and regenerated retinas. Bottom, $X-Z$ views of selected, traced BPs. Bottom ( $y$-axis) views of axon-branching patterns are color coded by heat maps to demonstrate relative numbers of axonal branches that cross circles of increasing distance from the initial branch point. Hotter colors represent more axons and cooler colors represent fewer axons.

ing two separate dendritic trees (Fig. $11 B$, last neuron) and the few nyx:: $m Y_{F P}{ }^{+}$cells displaced in the GCL (Fig. $1 M$, inset).

\section{ERG recordings reveal functional recovery of photoreceptor- bipolar connectivity in regenerated retinas}

To determine the extent of functional loss and recovery within regenerated zebrafish retinas, we measured ERG responses. ERG measurements were made in adult zebrafish after ouabain injections both at 3 DPI and at 80 DPI. ERG responses in the ouabaininjected eyes were compared with the contralateral, noninjected control eyes. The variability observed for control b-wave amplitudes likely reflects biological fish-fish variation rather than variations in recording conditions (such as electrode placement or light stimulus), as shown previously by Makhankov et al. (2004). At 3 DPI, a time point characterized by extensive damage and cell
Table 4. Sholl analysis (linear) of axons of nyx::mYFP bipolar neurons in control and regenerated retinas

\begin{tabular}{llrl}
\hline Parameter & $\begin{array}{l}p \text {-value (control } \\
\text { vs 60 DPI) }\end{array}$ & \multicolumn{1}{c}{$\chi^{2}$ or $t$} & $\begin{array}{l}\text { Statistical } \\
\text { test }^{a}\end{array}$ \\
\hline Maximum no. of intersections & 0.009 & $\chi_{(1)}^{2}=6.846$ & $\mathrm{~A}$ \\
Sum of intersections & 0.118 & $\chi_{(1)}^{2}=2.446$ & $\mathrm{~A}$ \\
Radius at maximum no. of intersections & 0.385 & $\chi_{(1)}^{2}=0.755$ & $\mathrm{~A}$ \\
Critical-value & 0.231 & $t_{(19.864)}=-1.243$ & $\mathrm{~B}$ \\
Radius of critical-value & 0.529 & $\chi_{(1)}^{2}=0.532$ & $\mathrm{~A}$ \\
Enclosing radius & 0.774 & $t_{(26.264)}=-0.334$ & $\mathrm{~B}$ \\
Kurtosis of the sampled data & 0.578 & $\chi_{(1)}^{2}=0.459$ & $\mathrm{~A}$ \\
Kurtosis of the fitted data & 0.578 & $\chi_{(1)}^{2}=0.237$ & $\mathrm{~A}$ \\
Skewness of the sampled data & 0.906 & $t_{(26.24)}=-0.117$ & $\mathrm{~B}$ \\
Skewness of the fitted data & 0.110 & $t_{(31.118)}=1.885$ & $\mathrm{~B}$ \\
\hline
\end{tabular}

${ }^{a} \mathrm{~A}$, Kruskal-Wallis; $\mathrm{B}$, Welch's two-sample $t$ test.

loss within inner retinal layers (Fig. 1), the ERG response induced by a $500 \mathrm{~ms}$ light flash showed an attenuated and abnormal waveform, even at saturating light stimulation levels, with defined a-wave activity but little or no recognizable b-wave component (Fig. 12A). The presence of an a-wave confirms the activity of intact photoreceptors spared from damage by the low concentration of ouabain that was injected, indicating that, not only do photoreceptors survive the lesions used in this study, they also remain functional. The loss of the b-wave confirms the lack of BP function. After regeneration, the ouabain-injected eyes displayed ERG responses that resembled the responses elicited in control eyes, but with a reduced overall magnitude (Fig. 12A). The recovery of the maximum b-wave amplitude in regenerated eyes, elicited by a saturating light flash, was $\sim 35 \%$ of control responses in the nondamaged eyes (Fig. 12B). To compare the "shape" of the damaged, regenerated, and control ERG waveforms independent of amplitude, we normalized the maximum amplitude of ERG responses during a saturating light flash. We observed a strong correlation between the b-wave features of regenerated and control retinas, which was lacking for responses elicited from freshly damaged retinas (Fig. 12C). We assessed the similarity of the regenerated versus freshly damaged ERG responses to the grand average of control retina responses using a sum of squares analysis (Fig. 12D). The sum of the squared deviations of the ERG waveforms quantifies the variation of the damaged/regenerated responses from control responses. For regenerated retinas, the ERG responses during light stimulation, dominated by the b-wave component, did not deviate significantly from control retina response ( $p=0.96, t$ test, $n=9,4)$. Furthermore, the light intensity-response relationship for b-waves elicited in regenerated retinas, when scaled to the maximum response of control retinas, demonstrated that, whereas b-wave amplitudes were reduced, the sensitivity of regenerated retinas closely matched that of control retinas (Fig. 12E). Overall, ERG responses in regenerated retinas confirm restoration of visual function, particularly connectivity between photoreceptors and BP neurons. These findings are in agreement with the structural evidence demonstrating restoration of normal $\mathrm{BP}$ dendritic morphologies and connectivities but reduced overall numbers of BPs.

\section{Discussion}

We provide the first detailed quantitative and qualitative report of BP morphologies, predicted circuitry, and physiology in regenerated adult zebrafish retina after destruction of multiple neuronal types. The major conclusions are as follows: (1) morphologies and complexities of dendritic trees of regenerated nyx:: $m$ YFP BP neurons are predominantly similar to those within 

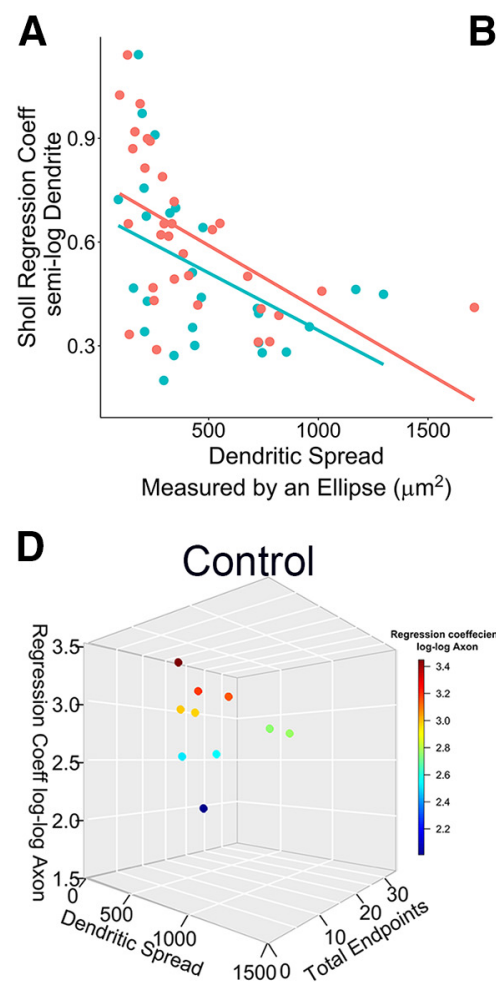

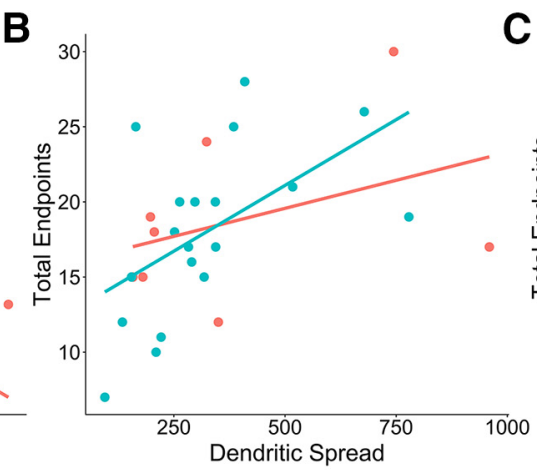

Measured by an Ellipse $\left(\mu \mathrm{m}^{2}\right)$

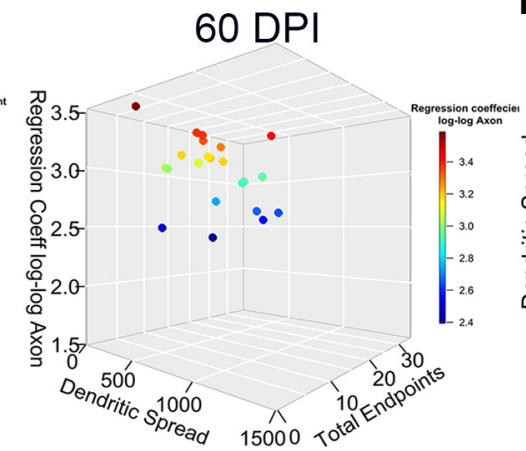

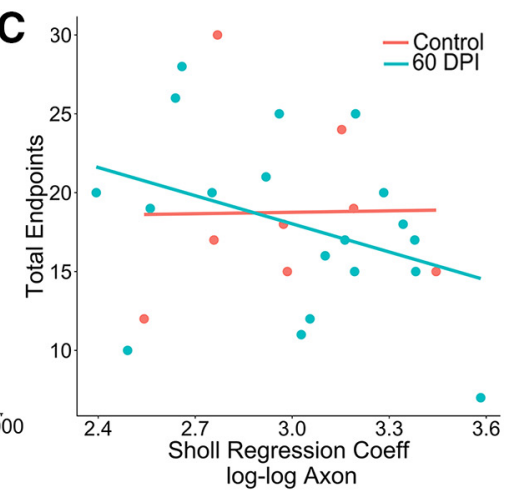

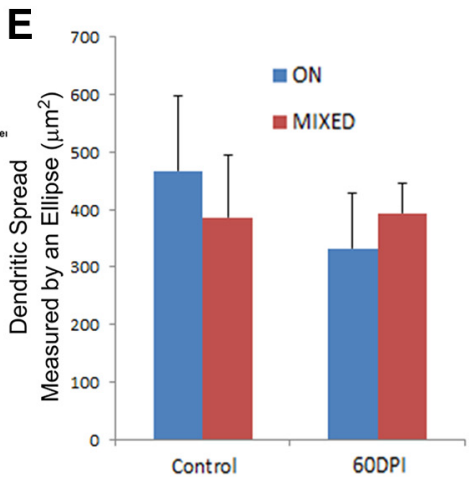

Figure 10. Correlations of morphometric parameters of control and regenerated nyx::mYFPBPs. $\boldsymbol{A}$, Dendritic complexity (Sholl regression coefficient, semi-log) is not differentially correlated with dendritic spread (ellipse method) in control versus regenerated BPs. Controls: $R^{2}=0.168 ; 60 \mathrm{DPI}, R^{2}=0.258\left(R^{2}\right.$ values for these graphs calculated by simple linear regression that does not account for individual eyes as blocking factors). $B$, Number of dendritic end points is not differentially correlated with dendritic spread in control versus regenerated BPs. Controls: $R^{2}=0.00 ; 60 \mathrm{DPI}, R^{2}=$ 0.159 . C, Number of dendritic end points is not differentially correlated with axon complexity (Sholl regression coefficient, log-log) in control versus regenerated BPs. Controls: $R^{2}=0.124 ; 60 \mathrm{DPI}$, $R^{2}=0.006$. D Visualization of three morphological parameters of BPs in control retinas and in regenerated retinas at $60 \mathrm{DPI}$ shows that the two BP populations occupy similar regions of the $3 D$ space. $E$, Dendritic spreads of BPs classified as $0 \mathrm{~N}$ or mixed ON/OFF. Dendritic spread is not predictive of this classification in control or regenerated retinas. Controls: $0 \mathrm{~N}$ versus mixed $p=0.690 ; 60$ $\mathrm{DPI}, 0 \mathrm{~N}$ versus mixed $p=0.643$.

Table 5. Pairwise analysis of continuous variables representing dendrite and axon morphologies of nyx::mYFP bipolar neurons in control and regenerated retinas

\begin{tabular}{|c|c|c|c|c|}
\hline Variable 1 & Variable 2 & $p$-value ${ }^{a}$ & $x^{2}$ & No. of eyes ${ }^{b}$ \\
\hline Dendritic field size (ellipse) & Dendritic complexity (Sholl regression, semi-log) & 0.729 & $\chi_{(1)}^{2}=0.121$ & 7,8 \\
\hline Dendritic field size (polygon) & Dendritic complexity (Sholl regression, semi-log) & 1 & $\chi_{(1)}^{2}=0$ & 7,8 \\
\hline Dendritic field size (ellipse) & Max intersections of dendrites (Sholl linear) & 0.643 & $\chi_{(1)}^{2}=0.214$ & 7,8 \\
\hline Dendritic complexity (Sholl regression, semi-log) & Axon complexity (Sholl regression, log-log) & 1 & $\chi_{(1)}^{2}=0$ & 3,6 \\
\hline Total dendritic endpoints & Axon complexity (Sholl regression, log-log) & 1 & $\chi_{(1)}^{2}=0$ & 3,6 \\
\hline Dendritic field size (ellipse) & Axon complexity (Sholl regression, log-log) & 0.121 & $\chi_{(1)}^{2}=2.4$ & 3,6 \\
\hline
\end{tabular}

${ }^{a}$ Kruskal-Wallis rank sum test of regression coefficients.

${ }^{b}$ Control, $60 \mathrm{DPI}$.

undamaged retina; (2) cone connectomes of regenerated BP neurons are largely restored; (3) axon terminal stratification and branching patterns of regenerated nyx::mYFP BPs show only minor differences compared with those within undamaged retina; (4) nyx::mYFP BPs in regenerated retina are reduced and variable in numbers, but show morphological diversity, suggesting a lack of bias during regeneration to favor any one BP morphology; and (5) a normal ERG waveform, but not amplitude, is restored in regenerated retina. These findings demonstrate that the regenerated retina of adult zebrafish has the underlying anatomical capacity to support retinal function.

\section{Morphologies and complexities of dendritic trees of regenerated BPs are restored}

The lesion used here destroyed inner retinal neurons while sparing photoreceptors and glia (Fimbel et al., 2007; Nagashima et al., 2013; Sherpa et al., 2014; Fig. 1), creating a disrupted retinal environment that could be considered challenging for regenerating BPs to elaborate their dendritic processes. However, when sampled at $60 \mathrm{DPI}$, when visually mediated behaviors are restored (Sherpa et al., 2014), BPs displayed dendritic field characteristics that were statistically undistinguishable from their undamaged counterparts, with the notable exception of one neuron displaying two dendritic trees. Similarly, dendritic territories of regenerated $x f z 43$ BPs were also restored after their cell-selective ablation in larval zebrafish, with the exception of regenerated ON-T1 xfz43 BPs, which had much larger dendritic trees (D'Orazi et al., 2016). The present study demonstrates that dendritic field sizes and complexities are restored by adult regenerated nyx::mYFP BPs and that this restoration can take place in a disturbed retinal environment. In goldfish subjected to a surgical retinal lesion, dendritic trees of regenerated RGCs also attained normal structural and modeled electrotonic properties (Cameron et al., 1999). Collectively, these results indicate that, even within a disrupted envi- 

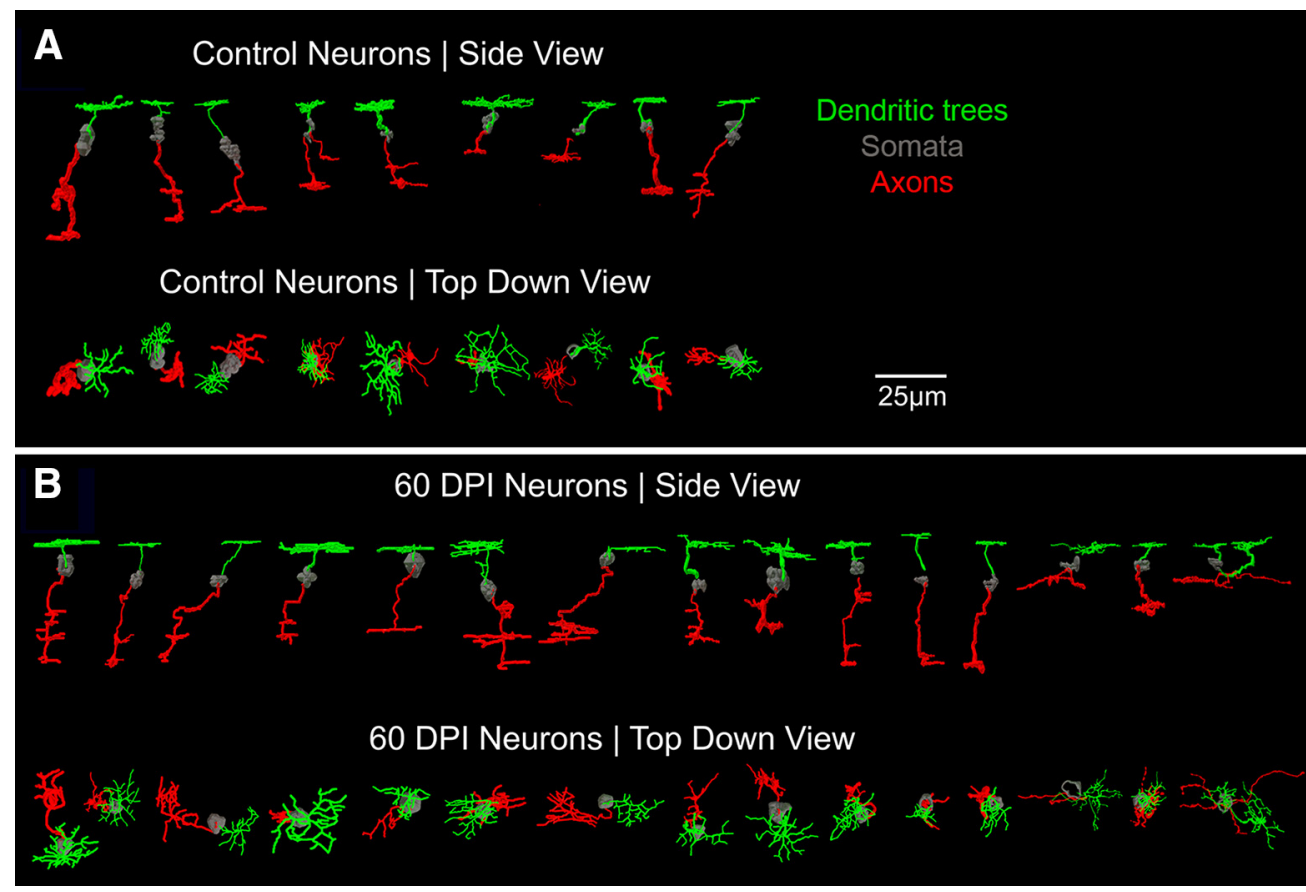

Figure 11. Surface renderings of control $(\boldsymbol{A})$ and regenerated $(\boldsymbol{B})$ nyx::mYFP BPs. The last three neurons in $\boldsymbol{B}$ were derived from a BrdU incorporation experiment and were BrdU ${ }^{+}$. Neurons are the same as the examples shown in Figure $8, A$ and $B$, and in the same order, except the neuron displaying two dendritic trees is also shown here.

ronment, regenerating neurons elaborate dendrites capable of adequately sampling the visual field and supporting retinal processing.

\section{Cone connectomes of regenerated BPs are largely restored}

The present study shows that dendrites of regenerated nyx::mYFP BPs collect synaptic input from multiple cone types, with distributions of connectivities similar to those within undamaged retinas. These include connections to SWS2 ("blue"), RH2 ("green"), and LWS ("red") cones. In addition, regenerated $\mathrm{PKC} \alpha{ }^{+}$BPs restored connections to SWS1 (UV) cones and double cones. Similarly, the ultrastructure of regenerated goldfish photoreceptor-BP synapses was verified as normal, with presynaptic ribbons and postsynaptic processes (Hitchcock and Cirenza, 1994) and amacrine cells within regenerated goldfish retina functionally integrated with surrounding, undamaged retina (Hitchcock, 1997). In the current study, retinal BPs were challenged to integrate accurately with undamaged photoreceptors and our anatomical and physiological measures of this integration showed that regenerated cone-BP circuitry supported retinal function.

After a cell-selective, cone-lesioning approach in larval zebrafish, undamaged H3-type horizontal cells successfully reconnected to regenerated UV cones, except when UV cone regeneration was delayed (Yoshimatsu et al., 2016). In the current study, regeneration of inner retinal neurons was allowed to follow a normal time course and regenerated BPs anatomically and physiologically rewired with photoreceptors. It will be important to determine whether delay of $\mathrm{BP}$ regeneration also results in faulty rewiring in adult retinas and if rewiring is accurate in the more highly disrupted environment of retina after a lesion that destroys all neurons and spares Müller glia (Sherpa et al., 2008). After this more extensive damage, the cone mosaic is disorganized (Stenkamp et al., 2001; Sherpa et al., 2008) and may represent an obstacle for restoration of cone-BP circuitry.

Also in larval zebrafish retina, regenerated $x f z 43$ BPs were challenged to form circuits with undamaged photoreceptors after cell-selective destruction of only this BP population (D'Orazi et al., 2016). Subsets of these BPs, the ON-T2 type and the OFF type, showed a slightly reduced bias toward synapsing with LWS cones after regeneration, instead favoring $\mathrm{RH} 2$ cones or other photoreceptor types (D'Orazi et al., 2016). Our findings of no differential biases in connectivity of regenerated nyx::mYFP BPs suggest that restoration of accurate connectivity patterns may be facilitated by a more robust regeneration process. Alternatively, the photoreceptors of mature zebrafish retina may be less plastic and less likely to make connectivity errors than those of growing larval retina, in which BP differentiation may not be complete. Finally, the differential bias observed previously (D'Orazi et al., 2016) may be specific for the ON-T2 and OFF type BP subpopulations and/or the bias may have been related to insufficient time ( $5 \mathrm{~d}$ ) for complete regeneration.

\section{Axon terminal stratification and branching patterns of regenerated $\mathrm{BPs}$ are largely restored}

The damage mode used here provided regenerating BPs with undamaged upstream synaptic partners (photoreceptors) and with downstream synaptic partners (amacrine cells and RGCs) that were themselves regenerating or had regenerated. Furthermore, prior studies of regenerated teleost fish retinas have documented the presence of histological abnormalities in the IPL of regenerated retina, specifically "laminar fusions," misplaced cell bodies bridging the INL and GCL (Hitchcock et al., 1992; Sherpa et al., 2008; Sherpa et al., 2014; examples in Fig. 8B). In the present study, we also observed reduced thickness of the IPL. Given these histological disruptions, it was somewhat surprising that the stratification patterns and Sholl regression coefficients of axons of regenerated nyx::mYFP BPs were similar to those in undamaged retinas.

The nyx::mYFP BP population was characterized previously as ON BPs based upon axon terminal stratifications in the deep IPL layers in larvae (Bahadori et al., 2006; Schroeter et al., 2006). However, in adult zebrafish, this population is clearly more het- 

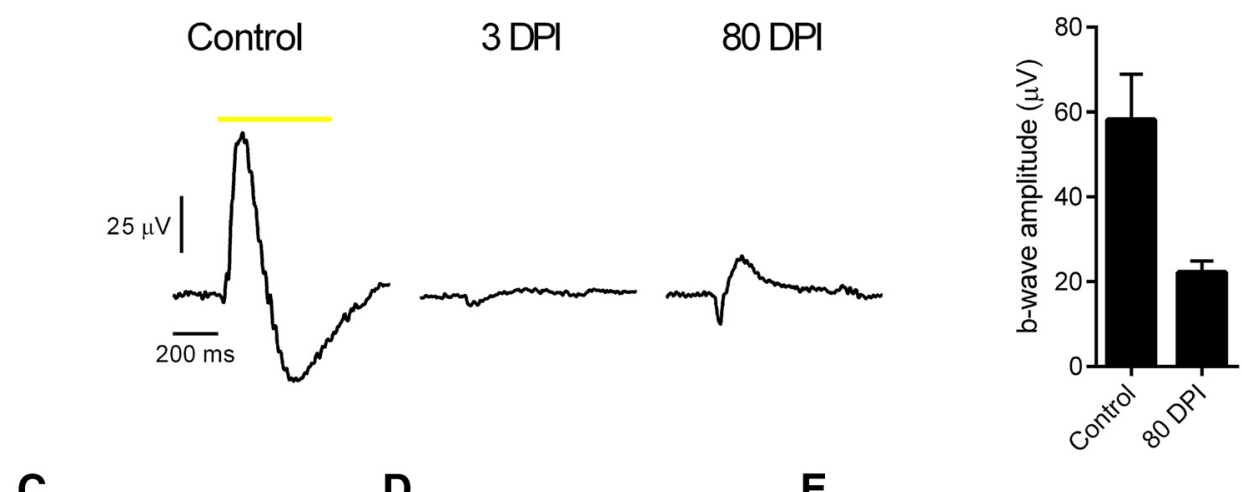

C
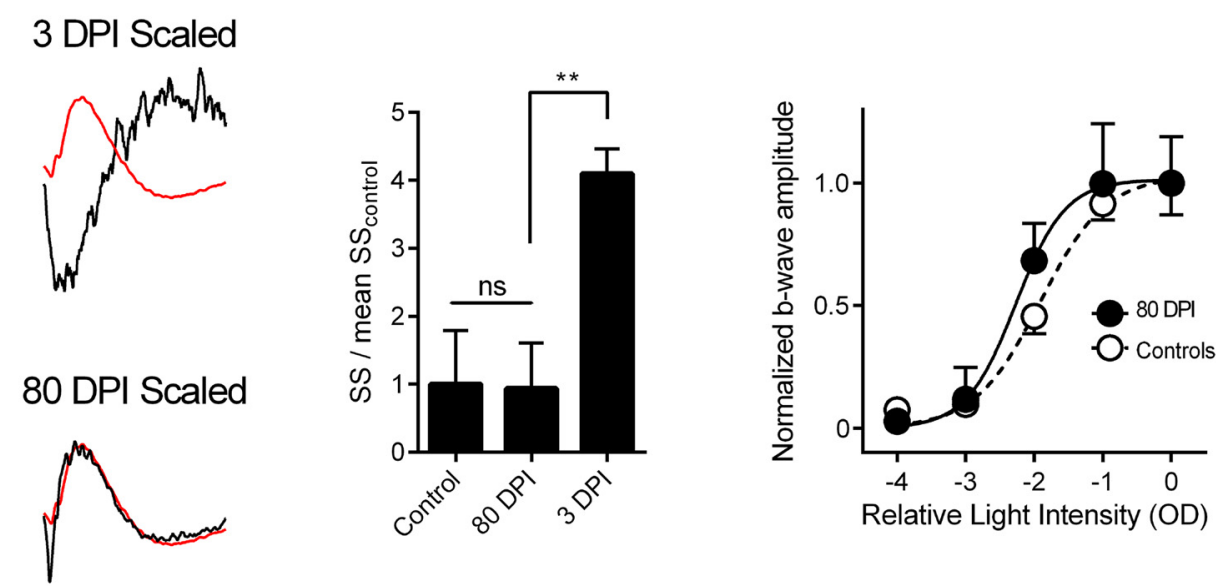

Figure 12. Regenerated retinas display near normal ERG responses that are absent in freshly damaged retinas. A, Representative ERG traces elicited by a $500 \mathrm{~ms} \mathrm{saturating} \mathrm{light} \mathrm{flash} \mathrm{for} \mathrm{freshly}$ damaged retina at $3 \mathrm{DPI}$ (middle) and regenerated retina at $80 \mathrm{DPI}$ (right) compared with ERG measurements made from an undamaged control retina (left). The control trace was made from the contralateral eye of the same fish from which the $80 \mathrm{DPI}$ trace was recorded. B, Mean b-wave amplitudes for control and $80 \mathrm{DPI}$ fish ( \pm SEM; $n=9$ and $n=4$, respectively). $\boldsymbol{C}$, Scaled representative ERG traces for the $3 \mathrm{DPI}$ fish (left, black) and the $80 \mathrm{DPI}$ fish (right, black). Each trace was scaled to the amplitude of the grand average of the control ERGs (red). D, Mean of the sum-of-squared deviations (SS) from the control grand average for the $80 \mathrm{DPI}, 3 \mathrm{DPl}$ and control groups ( \pm SEM). SS for each group were normalized to the mean SS for the controls (SS control). The $3 \mathrm{DPI}$ fish exhibited a significantly increased $S S\left({ }^{* *} p=0.0032, t\right.$ test, $\left.n=9,5\right)$ whereas the $S S$ for the $80 \mathrm{DPI}$ fish was indistinguishable from controls $(p=0.96, t$ test, $n=9,4) . \boldsymbol{E}$, Mean $b$-wave amplitudes over log light intensity for control (open circles) and $80 \mathrm{DPI}$ (closed circles) fish. Data fit with the Naka-Rushton function with the following best-fit parameters (with $95 \%$ confidence interval in parentheses): control (hashed line), $\mathrm{K}=-1.9 \mathrm{OD}$ ( -2.3 to -1.6$), n=0.91$ (0.33 to 1.5); $80 \mathrm{DPI}$ (solid line), $\mathrm{K}=-2.3 \mathrm{OD}(-2.8$ to -1.7$), n=1.2(-0.31$ to 2.7).

erogeneous, with some showing mixed ON/OFF stratification patterns (axon terminals in both OFF and ON layers), and monostratification, bi-stratification, or tri-stratification patterns. The axon-branching patterns of nyx:: $m Y F P$ BPs in adult zebrafish also appeared complex compared with the more simple terminals documented in larvae (Schroeter et al., 2006; D'Orazi et al., 2016). BP axon stratification and branching patterns were reconstituted in retinas that regenerated after lesioning of inner retinal neurons and there were no significant biases in any stratification pattern. These findings suggest that regenerating BPs deployed stratification programs that resulted in the adult patterns rather than recapitulation of the larval patterns. This is in contrast to a study in goldfish (Hitchcock and Cirenza, 1994), in which the depth distributions of synapses within the IPL were found to be distinct in regenerated retina (after a surgical lesion) compared with controls. These differences may be related to a BP subpopulation that was not sampled here. Alternatively, regeneration after a surgical lesion may result in more synaptogenesis errors than regeneration after damage of only inner retinal neurons. Cell-specific identification of postsynaptic partners of regenerated BPs is needed to further determine the accuracy of rewiring within the IPL.

\section{Morphological diversity of regenerated nyx::mYFP BPs is restored}

The complex visual processing functions of the retina require the activities of a diverse array of neurons and retinal BPs are excellent examples of structurally and functionally diverse retinal neurons (Connaughton et al., 2004; Li et al., 2012). The nyx::mYFP $\mathrm{BPs}$ represent this diversity well, with a variety of dendritic morphologies and axon stratification patterns. Remarkably, this range of morphologies, and combinations thereof, were restored in regenerated retina. The retinal regeneration process in the adult zebrafish therefore not only restores visually mediated reflexes and simple behaviors (Sherpa et al., 2008; Sherpa et al., 2014) and the b-wave of the ERG (present study), but also likely regains the underlying circuitry to support more complex visual functions.

\section{Normal ERG waveforms indicate physiological restoration of photoreceptor-BP connectivity}

The normal topographic features of the ERG waveforms observed in regenerated retinas were consistent with functional recovery and reestablishment of connections between photoreceptors and regenerated BPs. Robust b-waves confirmed activation of ON-BPs postsynaptic to hyperpolarized cone 
photoreceptors. However, reduced amplitude of the light responses was evident in the regenerated retinas compared with controls. It is possible that the reduced density of BPs observed in the regenerated retinas may limit b-wave amplitudes and/or restoration of function in regenerated retinas may be underestimated due to imperfect structural alignment that might reduce vectorial coordination or cell-cell synchronization. For ouabaindamaged eyes at 3 DPI, the defective ERG waveform was dominated by the negative a-wave potential, presumably due to hyperpolarization of the undamaged photoreceptors. The minor late positive component observed during saturating light flashes with some freshly damaged retinas may represent c-wave activity due to intact RPE/photoreceptor interactions. A detectable but reduced b-wave was also observed 50-70 DPI after "comprehensive retinal destruction" by ouabain in the goldfish (Mensinger and Powers, 1999). The present study now demonstrates this functional restoration in the zebrafish, the model organism available for more rapidly revealing the molecular genetic mechanisms for retinal regeneration (Ail and Perron, 2017). Together with the microanatomical observations and quantification, the ERG results demonstrate profound loss of BP function in the damaged adult zebrafish retina and provide powerful evidence of successful functional restoration after regeneration.

Note Added in Proof: The stratification entry S4S5S6 was accidentally entered as the last line in Table 3 instead of the fifth line of the Early Release version published Nov. 13, 2017. Table 3 and associated elements of the text and Fig. 10E have now been corrected.

\section{References}

Ahmad I, Del Debbio CB, Das AV, Parameswaran S (2011) Müller glia: a promising target for therapeutic regeneration. Invest Ophthalmol Vis Sci 52:5758-5764. CrossRef Medline

Ail D, Perron M (2017) Retinal degeneration and regeneration-lessons from fishes and amphibians. Curr Pathobiol Rep 5:67-78. CrossRef Medline

Allison WT, Barthel LK, Skebo KM, Takechi M, Kawamura S, Raymond PA (2010) Ontogeny of cone photoreceptor mosaics in zebrafish. J Comp Neurol 518:4182-4195. CrossRef Medline

Bahadori R, Biehlmaier O, Zeitz C, Labhart T, Makhankov YV, Forster U, Gesemann M, Berger W, Neuhauss SC (2006) Nyctalopin is essential for synaptic transmission in the cone dominated zebrafish retina. Eur J Neurosci 24:1664-1674. CrossRef Medline

Bernardos RL, Barthel LK, Meyers JR, Raymond PA (2007) Late-stage neuronal progenitors in the retina are radial Müller glia that function as retinal stem cells. J Neurosci 27:7028-7040. CrossRef Medline

Cameron DA, Vafai H, White JA (1999) Analysis of dendritic arbors of native and regenerated ganglion cells in the goldfish retina. Vis Neurosci 16:253-261. Medline

Connaughton VP, Nelson R (2000) Axonal stratification patterns and glutamate-gated conductance mechanisms in zebrafish retinal bipolar cells. J Physiol 524:135-146. CrossRef Medline

Connaughton VP, Graham D, Nelson R (2004) Identification and morphological classification of horizontal, bipolar, and amacrine cells within the zebrafish retina. J Comp Neurol 477:371-385. CrossRef Medline

D’Orazi FD, Zhao XF, Wong RO, Yoshimatsu T (2016) Mismatch of synaptic patterns between neurons produced in regeneration and during development of the vertebrate retina. Curr Biol 26:2268-2279. CrossRef Medline

Fausett BV, Goldman D (2006) A role for alpha1 tubulin-expressing Müller glia in regeneration of the injured zebrafish retina. J Neurosci 26:63036313. CrossRef Medline

Ferreira TA, Blackman AV, Oyrer J, Jayabal S, Chung AJ, Watt AJ, Sjöström PJ, van Meyel DJ (2014) Neuronal morphometry directly from bitmap images. Nat Methods 11:982-984. CrossRef Medline

Fimbel SM, Montgomery JE, Burket CT, Hyde DR (2007) Regeneration of inner retinal neurons after intravitreal injection of ouabain in zebrafish. J Neurosci 27:1712-1724. CrossRef Medline

Fraser B, DuVal MG, Wang H, Allison WT (2013) Regeneration of cone photoreceptors when cell ablation is primarily restricted to a particular cone subtype. PLoS One 8:e55410. CrossRef Medline

Gorsuch RA, Hyde DR (2014) Regulation of Müller glial dependent neuronal regeneration in the damaged adult zebrafish retina. Exp Eye Res 123: 131-140. CrossRef Medline

Hamon A, Roger JE, Yang XJ, Perron M (2016) Müller glial cell-dependent regeneration of the neural retina: an overview across vertebrate model systems. Dev Dyn 245:727-738. CrossRef Medline

Hitchcock PF (1997) Tracer coupling among regenerated amacrine cells in the retina of the goldfish. Vis Neurosci 14:463-472. CrossRef Medline

Hitchcock PF, Cirenza P (1994) Synaptic organization of regenerated retina in the goldfish. J Comp Neurol 343:609-616. Medline

Hitchcock PF, Lindsey Myhr KJ, Easter SS Jr, Mangione-Smith R, Jones DD (1992) Local regeneration in the retina of the goldfish. J Neurobiol 23: 187-203. CrossRef Medline

Hyde DR, Reh TA (2014) The past, present, and future of retinal regeneration. Exp Eye Res 123:105-106. CrossRef Medline

Jorstad NL, Wilken MS, Grimes WN, Wohl SG, VandenBosch LS, Yoshimatsu T, Wong RO, Rieke F, Reh TA (2017) Stimulation of functional neuronal regeneration from Müller glia in adult mice. Nature 548:103107. CrossRef Medline

Karl MO, Hayes S, Nelson BR, Tan K, Buckingham B, Reh TA (2008) Stimulation of neural regeneration in the mouse retina. Proc Natl Acad Sci U S A 105:19508-19513. CrossRef Medline

Kraft R, Escobar MM, Narro ML, Kurtis JL, Efrat A, Barnard K, Restifo LL (2006) Phenotypes of Drosophila brain neurons in primary culture reveal a role for fascin in neurite shape and trajectory. J Neurosci 26:8734-8747. CrossRef Medline

Lenkowski JR, Raymond PA (2014) Müller glia: Stem cells for generation and regeneration of retinal neurons in teleost fish. Prog Retin Eye Res 40:94-123. CrossRef Medline

Li YN, Tsujimura T, Kawamura S, Dowling JE (2012) Bipolar cellphotoreceptor connectivity in the zebrafish (Danio rerio) retina. J Comp Neurol 520:3786-3802. CrossRef Medline

Longair MH, Baker DA, Armstrong JD (2011) Simple Neurite Tracer: open source software for reconstruction, visualization and analysis of neuronal processes. Bioinformatics 27:2453-2454. CrossRef Medline

Maier W, Wolburg H (1979) Regeneration of the goldfish retina after exposure to different doses of ouabain. Cell Tissue Res 202:99-118. Medline

Makhankov YV, Rinner O, Neuhauss SC (2004) An inexpensive device for non-invasive electroretinography in small aquatic vertebrates. J Neurosci Methods 135:205-210. CrossRef Medline

Mensinger AF, Powers MK (1999) Visual function in regenerating teleost retina following cytotoxic lesioning. Vis Neurosci 16:241-251. Medline

Nagashima M, Barthel LK, Raymond PA (2013) A self-renewing division of zebrafish Müller glial cells generates neuronal progenitors that require $\mathrm{N}$-cadherin to regenerate retinal neurons. Development 140:4510-4521. CrossRef Medline

Powell C, Cornblath E, Elsaeidi F, Wan J, Goldman D (2016) Zebrafish Müller glia-derived progenitors are multipotent, exhibit proliferative biases and regenerate excess neurons. Sci Rep 6:24851. CrossRef Medline

Renninger SL, Gesemann M, Neuhauss SC (2011) Cone arrestin confers cone vision of high temporal resolution in zebrafish larvae. Eur J Neurosci 33:658-667. CrossRef Medline

Saade CJ, Alvarez-Delfin K, Fadool JM (2013) Rod photoreceptors protect from cone degeneration-induced retinal remodeling and restore visual responses in zebrafish. J Neurosci 33:1804-1814. CrossRef Medline

Salbreux G, Barthel LK, Raymond PA, Lubensky DK (2012) Coupling mechanical deformations and planar cell polarity to create regular patterns in the zebrafish retina. PLoS Comput Biol 8:e1002618. CrossRef Medline

Schindelin J, Arganda-Carreras I, Frise E, Kaynig V, Longair M, Pietzsch T, Preibisch S, Rueden C, Saalfeld S, Schmid B, Tinevez JY, White DJ, Hartenstein V, Eliceiri K, Tomancak P, Cardona A (2012) Fiji: an opensource platform for biological-image analysis. Nat Methods 9:676-682. CrossRef Medline

Schroeter EH, Wong RO, Gregg RG (2006) In vivo development of retinal ON-bipolar cell axonal terminals visualized in nyx::MYFP transgenic zebrafish. Vis Neurosci 23:833-843. CrossRef Medline

Sherpa T, Fimbel SM, Mallory DE, Maaswinkel H, Spritzer SD, Sand JA, Li L, Hyde DR, Stenkamp DL (2008) Ganglion cell regeneration following whole-retina destruction in zebrafish. Dev Neurobiol 68:166-181. CrossRef Medline 
Sherpa T, Hunter SS, Frey RA, Robison BD, Stenkamp DL (2011) Retinal proliferation response in the buphthalmic zebrafish, bugeye. Exp Eye Res 93:424-436. CrossRef Medline

Sherpa T, Lankford T, McGinn TE, Hunter SS, Frey RA, Sun C, Ryan M, Robison BD, Stenkamp DL (2014) Retinal regeneration is facilitated by the presence of surviving neurons. Dev Neurobiol 74:851-876. CrossRef

Sholl DA (1953) Dendritic organization in the neurons of the visual and motor cortices of the cat. J Anat 87:387-406. Medline

Stenkamp DL (2007) Neurogenesis in the fish retina. Int Rev Cytol 259:173224. CrossRef Medline

Stenkamp DL, Powers MK, Carney LH, Cameron DA (2001) Evidence for two distinct mechanisms of neurogenesis and cellular pattern formation in regenerated goldfish retinas. J Comp Neurol 431:363-381. CrossRef Medline

Suzuki S, Kaneko A (1990) Identification of bipolar cell subtypes by protein kinase C-like immunoreactivity in the goldfish retina. Vis Neurosci 5:223-230. CrossRef Medline

Takechi M, Hamaoka T, Kawamura S (2003) Fluorescence visualization of ultraviolet-sensitive cone photoreceptor development in living zebrafish. FEBS Lett 553:90-94. CrossRef Medline

Ueki Y, Wilken MS, Cox KE, Chipman L, Jorstad N, Sternhagen K, Simic M, Ullom K, Nakafuku M, Reh TA (2015) Transgenic expression of the proneural transcription factor Ascll in Müller glia stimulates retinal regeneration in young mice. Proc Natl Acad Sci U S A 112:13717-13722. CrossRef Medline

Wan J, Goldman D (2016) Retina regeneration in zebrafish. Curr Opin Genet Dev 40:41-47. CrossRef Medline

Westerfield M (2007) The Zebrafish Book; A guide for the laboratory use of zebrafish (Danio rerio). Eugene, OR: University of Oregon.

Yazulla S, Studholme KM (2001) Neurochemical anatomy of the zebrafish retina as determined by immunocytochemistry. J Neurocytol 30:551-592. CrossRef Medline

Yoshimatsu T, D’Orazi FD, Gamlin CR, Suzuki SC, Suli A, Kimelman D, Raible DW, Wong RO (2016) Presynaptic partner selection during retinal circuit reassembly varies with timing of neuronal regeneration in vivo. Nat Commun 7:10590. CrossRef Medline 